\title{
BILLIARD DYNAMICS OF EINSTEIN-MATTER SYSTEMS NEAR A SPACELIKE SINGULARITY
}

Thibault DAMOUR, Marc HENNEAUX and Hermann NICOLAI

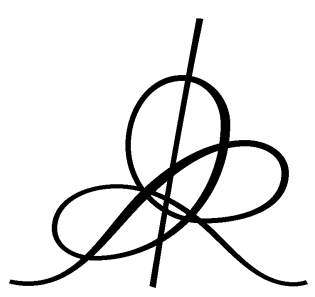

Institut des Hautes Études Scientifiques 35 , route de Chartres 91440 - Bures-sur-Yvette (France)

Octobre 2002

IHES $/ \mathrm{P} / 02 / 72$

To appear in the Proceedings of the "School on Quantum Gravity", held in Valdivia in January 2002, A. Gomberoff and D. Marolf eds., Kluwer Academic/Plenum Press, New York. 


\title{
Billiard dynamics of Einstein-matter systems near a spacelike singularity
}

\author{
Thibault Damour ${ }^{a}$, Marc Henneaux ${ }^{b, c}$ and Hermann \\ Nicolai $^{d}$
}

${ }^{a}$ Institut des Hautes Etudes Scientifiques, 35, Route de Chartres, F-91440 Bures-sur-Yvette, France

${ }^{b}$ Physique Théorique et Mathématique, Université Libre de Bruxelles, C.P. 231, B-1050, Bruxelles, Belgium

${ }^{c}$ Centro de Estudios Científicos, Casilla 1469, Valdivia, Chile

${ }^{d}$ Max-Planck-Institut für Gravitationsphysik, Albert-Einstein-Institut, Am Mühlenberg 1, D-14476 Golm, Germany

\begin{abstract}
It is shown in detail that the dynamics in the vicinity of a spacelike singularity of the $D$-dimentional Einstein-dilaton- $p$-form system can be described, at each spatial point, as a billiard motion in a region of hyperbolic space. This is done within the Hamiltonian formalism. A key rôle is played in the derivation by the Iwasawa decomposition of the spatial metric. We also comment on the strong coupling/small tension limit of the theory.
\end{abstract}

\footnotetext{
${ }^{1}$ To appear in the Proceedings of the "School on Quantum Gravity", held in Valdivia in January 2002, A. Gomberoff and D. Marolf eds., Kluwer Academic/Plenum Press, New York.
} 


\section{Introduction}

\subsection{BKL analysis in four and higher spacetime dimen- sions}

The non-linearities of the Einstein equations are notably known to prevent the construction of an exact, general solution. Only peculiar solutions, corresponding to idealized situations, have been explicitly derived. From this perspective, the work of Belinskii, Khalatnikov and Lifshitz [BKL70, BKL82] known as "BKL" -, which describes the asymptotic, general behaviour of the gravitational field in four spacetime dimensions as one approaches a spacelike singularity, appears to be quite remarkable. As argued by these authors, the spatial points decouple in this regime, in the sense that the dynamical evolution of the spatial metric at each spatial point is asymptotically governed by a set of ordinary differential equations with respect to time involving only the metric and its time derivatives at that point. These differential equations are the same as those that arise in some spatially homogeneous cosmological models, which provide therefore invaluable insight into the qualitative features of the general solution.

In the vacuum case, the spatially homogeneous models that capture the behaviour of the general solution are of "Bianchi types" IX or VIII (homogeneity groups equal to $S U(2)$ or $S L(2, R))$. The asymptotic evolution of the metric can then be pictured as an infinite sequence of oscillations of the scale factors along independent spatial directions [BKL70, BKL82]. This regime is called "oscillatory" or "of mixmaster type" [M69a] and exhibits strong chaotic features [LLK71, CB83]. The coupling to matter fields does not change the picture, except if one includes a massless scalar field (equivalent to a perfect fluid with "stiff" equation of state $p=\rho$ ), in which case the relevant homogeneity group is simply the abelian group of translations in $R^{3}$ ("Bianchi I" model). The chaotic evolution is replaced by a monotone expansion or contraction of the scale factors [BK73], mimicking at each spatial point the Kasner solution. This behaviour is called "Kasner-like".

It is natural to investigate whether the BKL analysis remains valid in higher dimensions. The study of this question was started some time ago in the context of pure gravity (with no symmetry assumption) in [DHS85, DHHST86], where it was shown that the general BKL ideas remain valid: spatial points decouple as one approaches a spacelike singularity, i.e., the dynamical evolution at each spatial point of the scale factors is again governed 
by ordinary differential equations. However, while the general behaviour of solutions of the vacuum Einstein equations remains oscillatory for spacetime dimensions $D \leq 10$, it ceases to be so for spacetime dimensions $D \geq 11$, where it becomes Kasner-like. Furthermore, just as in four spacetime dimensions, the coupling to a massless scalar field suppresses the chaotic behaviour in any number of spacetime dimensions (when it is present), and makes the solution monotone.

The authors of [DHS85] did not consider the inclusion of massless $p$-forms, which are part of the low-energy bosonic sector of superstring/M-theory models. This task was undertaken in [DH00a, DH00b], with the finding that these $p$-forms play a crucial role and can reinstate chaos when it is otherwise suppressed. In particular, even though pure gravity is non chaotic in $D=11$ spacetime dimensions, the 3-form of 11-dimensional supergravity renders the system chaotic. Similarly, the bosonic sectors of all ten-dimensional supergravities related to string models define chaotic dynamical systems, thanks again to the $p$-forms.

The best way to grasp the asymptotic behaviour of the fields as one approaches a spacelike singularity is based on the qualitative Hamiltonian methods initiated by Misner [M69b]. These naturally lead, in four dimensions, to a billiard description of the asymptotic evolution, in which the spatial scale factors define a geodesic motion in a region of the Lobachevsky plane $\mathrm{H}_{2}$, interrupted by geometric reflections against the walls bounding this region [C72, M94]. Chaos follows from the fact that the billiard has finite volume ${ }^{2}$.

It turns out that this useful billiard description is quite general and can be extended to higher spacetime dimensions, with $p$-forms and dilaton. If $d=D-1$ is the number of spatial dimensions, the billiard is a region of hyperbolic space $H_{d}$ in the presence of the dilaton, or $H_{d-1}$ if no dilaton is present. [If there are $k$ dilatons, the dimension of the relevant hyperbolic space is $d+k-1$.] Besides the dimension of the hyperbolic billiard, the other ingredients that enter its definition are the walls that bound it. These walls can be of different types [DH00a, DH01]: symmetry walls related to the offdiagonal components of the spatial metric, gravitational walls related to the spatial curvature, and $p$-form walls (electric and magnetic) arising from the $p$-form energy-density. All these walls are hyperplanes and the billiard is a

\footnotetext{
${ }^{2}$ Throughout this paper, the word billiard used as a noun in the singular will denote the dynamical system consisting of a ball moving freely on a "table" (region in some Riemannian space), with elastic bounces against the edges. Billiard will also sometimes mean the table itself.
} 
convex polyhedron with finitely many vertices, some of which are at infinity.

The fact that the asymptotic dynamics admits a billiard description was announced in [DH00a, DH01], where it was derived using the somewhat heuristic arguments standard in BKL-type discussions. The purpose of this paper is to provide a more complete derivation of the billiard picture, in the general (inhomogeneous) $D$-dimensional situation, with dilaton and $p$-form gauge fields. For that purpose, we shall rely on the Iwasawa decomposition (see e.g. [H78]) of the spatial metric. This provides an efficient derivation of the symmetry walls in any number of spacetime dimensions, which we do by working out explicitly the Hamiltonian that governs the dynamics in the "BKL" or "small volume" limit.

\subsection{Organization of the paper}

After fixing the conventions, we discuss in the next two sections the Kasner (= invariant under spatial translations) solution in $D$ spacetime dimensions, with a dilaton. This solution plays a particularly important rôle because it turns out to describe the free motion between collisions. First, we consider diagonal metrics and develop the geometry of the scale factors (section 2). Next, we do not assume that the spatial metric is diagonal and show, using the Iwasawa decomposition of the spatial metric, that the off-diagonal components induce "symmetry walls" in the BKL limit (section 3). In fact, many of the billiard ideas can already be introduced in this simple context.

In section 4, we derive the billiard picture in full generality, without imposing any homogeneity conditions on the metric and the matter fields. We show how the gravitational and $p$-form walls emerge (in addition to the symmetry walls) near a spacelike singularity when one takes the BKL limit. We also prove that Yang-Mills or Chern-Simons couplings do not affect the billiard picture.

In section 5, we relate the BKL limit to other limits that have been considered in the past ("strong coupling limit" [I76], "zero velocity of light limit" [T78]). We also discuss the connection with the so-called "velocitydominated" behavior [E72]. The last section (section 6) provides a brief conclusion as well as a short survey of some related developments (emergence of Kac-Moody algebras for specified models, lists of chaotic and non-chaotic models).

Four appendices illustrate and discuss further some aspects of the problem. Appendix A analyzes the Iwasawa decomposition and the asymptotics 
of the off-diagonal Kasner metric in the case of three spatial dimensions. Appendices B and C shed further light on the freezing off of the off-diagonal components and of the electromagnetic variables (in a sense made precise in the text) as one goes to the singularity. Finally, appendix D is devoted to the Hamiltonian reduction of the system in the general case.

We should stress that our analysis is purely classical and is accordingly, as it stands, valid only up to the Planck scale. We also drop the fermionic fields. It is reasonable to expect, however, that some of the ideas discussed here will remain relevant in a more general context, at least qualitatively.

The subject of Hamiltonian cosmology has a long history in the context of four-dimensional, spatially homogeneous spacetimes and provides useful insight on the general discussion presented here. Excellent reviews on this subject, with an extensive bibliography, are [R72b, RS75, J01].

\subsection{Models and Conventions}

We consider models of the general form

$$
\begin{aligned}
& S\left[g_{\mu \nu}, \phi, A^{(p)}\right]=\int d^{D} x \sqrt{-(D)} g\left[R-\partial_{\mu} \phi \partial^{\mu} \phi\right. \\
& \left.\quad-\frac{1}{2} \sum_{p} \frac{1}{(p+1) !} e^{\lambda^{(p)} \phi} F_{\mu_{1} \cdots \mu_{p+1}}^{(p)} F^{(p) \mu_{1} \cdots \mu_{p+1}}\right]+ \text { "more" }
\end{aligned}
$$

where we have chosen units such that $16 \pi G=1$. The spacetime dimension is left unspecified. We work (as a convenient common formulation) in the Einstein conformal frame, and we normalize the kinetic term of the "dilaton" $\phi$ with a weight 1 with respect to the Ricci scalar. The Einstein metric $g_{\mu \nu}$ has Lorentz signature $(-,+, \cdots,+)$ and is used to lower or raise the indices. Its determinant is ${ }^{(D)} g$, where the index $D$ is used to avoid any confusion with the determinant of the spatial metric introduced below. The integer $p \geq 0$ labels the various $p$-forms $A^{(p)}$ present in the theory, with field strengths $F^{(p)}$ equal to $d A^{(p)}$,

$$
F_{\mu_{1} \cdots \mu_{p+1}}^{(p)}=\partial_{\mu_{1}} A_{\mu_{2} \cdots \mu_{p+1}}^{(p)} \pm p \text { permutations . }
$$

In fact, the field strength could be modified by additional coupling terms of Yang-Mills or Chapline-Manton type [BRWN82, CM83] (e.g., $F_{C}=d C^{(2)}-$ $C^{(0)} d B^{(2)}$ for two 2-forms $C^{(2)}$ and $B^{(2)}$ and a 0 -form $C^{(0)}$, as it occurs in 
ten-dimensional type IIB supergravity), but we include these additional contributions to the action in "more". Similarly, "more" might contain ChernSimons terms, as in the action for eleven-dimensional supergravity [CJS78]. If there are many $p$-form gauge fields with same form degree $p$, we use different letters $A, B, C, \ldots$ to distinguish them, as we just did. The real parameter $\lambda^{(p)}$ measures the strength of the coupling to the dilaton. When $p=0$, we assume that $\lambda^{(0)} \neq 0$, so that there is only one dilaton. This is done mostly for notational convenience. If there were other dilatons among the 0 -forms, these should be separated off from the $p$-forms because they play a distinct rôle. They would appear as additional scale factors and would increase the dimensions of the relevant hyperbolic billiard (they define additional spacelike directions in the space of the scale factors: see Eq. (2.13) below, in which $(d \phi)^{2}$ should be replaced by $\sum_{i}\left(d \phi^{i}\right)^{2}$, where $i$ runs over the dilatons).

Our work applies both to past and future singularities. In particular, it applies to Schwarzschild-type of singularities inside black holes. To follow historical usage, we shall assume for definiteness that the spacelike singularity occurs in the past, at $t=0\left(t \rightarrow 0^{+}\right)$.

\section{Kasner solution - Diagonal case}

\subsection{Diagonal case}

One of the basic ingredients in the asymptotic description of the fields as one goes to the singularity is the Kasner solution (generalized to include the dilaton, in $D$ spacetime dimensions).

The Kasner solution is the general solution of the Einstein-dilaton equations (no $p$-form) in the spatially homogeneous context, where all the fields depend only on the time coordinate ("Bianchi I" models). Assuming the initial data (spatial metric and extrinsic curvature of initial slice) to be diagonal ("diagonal case"), one easily finds that the metric and the dilaton are given by

$$
\begin{aligned}
d s^{2} & =-d t^{2}+\sum_{i=1}^{d} a_{i}^{2}(t)\left(d x^{i}\right)^{2}, a_{i}(t)=t^{p^{i}} \\
\phi & =-p^{\phi} \ln t+C
\end{aligned}
$$


where $p^{i}, p^{\phi}$ and $C$ are integration constants subject to the conditions

$$
\sum_{i=1}^{d} p^{i}=1
$$

and

$$
\sum_{i=1}^{d}\left(p^{i}\right)^{2}-\left(\sum_{i=1}^{d} p^{i}\right)^{2}+\left(p^{\phi}\right)^{2}=0 .
$$

(the minus sign in front of $p^{\phi}$ in (2.2) is included for the sake of uniformity in the formulas below). The second condition is the Hamiltonian constraint $G_{00}-(1 / 2) T_{00}=0$. The $\left(p^{i}, p^{\phi}\right)$ are known as the Kasner exponents. In the absence of the dilaton, one must simply set $p^{\phi}$ (and $C$ ) equal to zero in the above expressions. In this latter case, there is at least one Kasner exponent $p^{i}$ that is negative, so that at least one of the scale factors $a_{i}$ blows up as $t \rightarrow 0$. In contrast, the scale factors associated with positive Kasner exponents monotonously contract to zero. In the presence of the dilaton, however, all the $p^{i}$ can be positive simultaneously. In all cases there is an overall contraction since the determinant $g$ of the spatial metric tends to zero,

$$
g=t^{2} \rightarrow 0 \text { for } t \rightarrow 0 .
$$

It is convenient to redefine the time coordinate $t$ as

$$
t \rightarrow \tau=-\ln t
$$

This puts the singularity $t \rightarrow 0^{+}$at $\tau \rightarrow+\infty$. Similarly, as $t \rightarrow+\infty$, we have $\tau \rightarrow-\infty$. In terms of $\tau$, the logarithms of the scale factors

$$
\beta^{i}=-\ln a_{i}
$$

and the dilaton behave linearly,

$$
\beta^{i}=p^{i} \tau, \quad \phi=p^{\phi} \tau .
$$

The Kasner exponents appear therefore as the $\tau$-time velocities of the $\beta^{i}, \phi$. 


\subsection{Geometry of the space of the scale factors}

\subsubsection{Supermetric and Hamiltonian}

The Kasner solution can be viewed as a null geodesic in the "superspace" coordinatized by the metric components $g_{i j}$ and by $\phi$. Indeed, the action that governs the time-evolution of spatially-homogeneous models of type I, which is obtained from the action (1.1) by setting $A^{(p)}=0$ and assuming the other fields to depend on time only, reads, in terms of an arbitrary reparametrized time $x^{0}=x^{0}(t)$,

$$
S\left[g_{i j}, \phi, \tilde{N}\right]=\int d x^{0} \frac{1}{\tilde{N}}\left[\frac{1}{4}\left(\operatorname{tr}\left(G^{-1} \dot{G}\right)^{2}-\left(\operatorname{tr} G^{-1} \dot{G}\right)^{2}\right)+\dot{\phi}^{2}\right]
$$

where we have set

$$
d s^{2}=-(\tilde{N} \sqrt{g})^{2}\left(d x^{0}\right)^{2}+g_{i j} d x^{i} d x^{j}
$$

and $\dot{F} \equiv d F / d x^{0}$. We have adopted matrix notations in which $G$ stands for the $d \times d$ matrix with matrix elements $\left(g_{i j}\right)$. The use of the rescaled lapse $\tilde{N}=N / \sqrt{g}$ makes the subsequent formulas simpler. We assume that $x^{0}$ increases as one goes to the singularity, i.e., we take the minus sign in the relation $d t=-\tilde{N} \sqrt{g} d x^{0}$ relating the proper time to $x^{0}$.

The action (2.9) is the action for a free particle with coordinates $\left(g_{i j}, \phi\right)$ moving in a curved space with metric

$$
d \sigma^{2}=\frac{1}{4}\left[\operatorname{tr}\left(G^{-1} d G\right)^{2}-\left(\operatorname{tr} G^{-1} d G\right)^{2}\right]+d \phi^{2}
$$

Observe that we designate by $d \sigma^{2}$ the line element in superspace to distinguish it from the line element in physical space time, which we denote by $d s^{2}$. The first two terms in the right-hand side of (2.11) define what is known as the De Witt supermetric in the space of the $g_{i j}$ 's, considered first in [D67]. Furthermore, because of time reparametrization invariance, the velocities are constrained by

$$
\frac{1}{4}\left(\operatorname{tr}\left(G^{-1} \dot{G}\right)^{2}-\left(\operatorname{tr} G^{-1} \dot{G}\right)^{2}\right)+\dot{\phi}^{2}=0
$$

as follows from extremizing (2.9) with respect to $\tilde{N}$. Thus, the motion is given by a null geodesic of the metric (2.11), as announced. 
For diagonal metrics, the supermetric (2.11) reduces to

$$
d \sigma^{2}=\operatorname{tr}(d \beta)^{2}-(\operatorname{tr} d \beta)^{2}+d \phi^{2}
$$

The action becomes therefore

$$
S\left[\beta^{\mu}, \tilde{N}\right]=\int d x^{0} \frac{1}{\tilde{N}} G_{\mu \nu} \dot{\beta}^{\mu} \dot{\beta}^{\nu}
$$

where we have used the notation $\beta^{0} \equiv \phi$ for the dilaton (if it is present) and defined $G_{\mu \nu}$ through $d \sigma^{2}=G_{\mu \nu} d \beta^{\mu} d \beta^{\nu}(\mu, \nu=0,1, \cdots, d$ with a dilaton, $\mu, \nu=1, \cdots, d$ without dilaton). It is natural to collect together the scale factors and the dilaton, since the dilaton can be viewed as a scale factor in one extra dimension. Although the metric (2.11) has a non-vanishing curvature, the metric (2.13) induced in the space of the scale factors (including the dilaton if any) is flat. The diagonal Kasner solution is thus just a lightlike straight line in the space of the scale factors. The Hamiltonian form of the action is

$$
S\left[\beta^{\mu}, \pi_{\mu}, \tilde{N}\right]=\int d x^{0}\left[\pi_{\mu} \dot{\beta}^{\mu}-\frac{\tilde{N}}{4} G^{\mu \nu} \pi_{\mu} \pi_{\nu}\right]
$$

with

$$
G^{\mu \nu} \pi_{\mu} \pi_{\nu} \equiv \sum_{i=1}^{d} \pi_{i}^{2}-\frac{1}{d-1}\left(\sum_{i=1}^{d} \pi_{i}\right)^{2}+\pi_{\phi}^{2}
$$

where $\left(\pi_{i}, \pi_{\phi}\right) \equiv\left(\pi_{\mu}\right)$ are the momenta respectively conjugate to $\beta^{i}$ and $\phi$, i.e., $\pi_{\mu}=2 \tilde{N}^{-1} G_{\mu \nu} \dot{\beta}^{\nu}$.

The metric $(2.13)$ has Minkowskian signature $(-,+,+, \cdots,+)$. The minus sign is due to the presence of gravity, and more specifically to the conformal factor in the metric; for the other fields, the kinetic term is always positive definite. This reflects the familiar fact that the gravitational action is not bounded from below (switching to Euclidean signature will not change the De Witt supermetric). Conformal transformations of the metric, in which the scale factors are all scaled in the same fashion, make $d \sigma^{2}$ negative. It is this characteristic feature of gravity which is responsible for the Lorentzian type of the Kac-Moody algebras which emerge in the analysis of the billiard symmetries [DH01]. The Lorentzian signature of the metric in the space of the scale factors enables one to define the light cone through any point. We define the time-orientation to be such that future-pointing vectors $v^{\mu}$ have $\sum_{i} v^{i}>0$. Small volumes (small $g$ ) mean large positive values of $\sum_{i} \beta^{i}$. In 
contrast, large volumes (large $g$ ) mean large negative values of $\sum_{i} \beta^{i}$. We are interested in the small volume limit.

\subsubsection{Hyperbolic space}

With the gauge choice $\tilde{N}=1$, the solution of the dynamical equations read

$$
\beta^{\mu}=v^{\mu} x^{0}+\beta_{0}^{\mu}
$$

while the constraint is

$$
G_{\mu \nu} v^{\mu} v^{\nu}=0
$$

It follows from $(2.18)$ that $\sum_{i} v^{i} \neq 0^{3}$. In fact, since $g \sim \exp \left[-2\left(\sum_{i} v^{i}\right) x^{0}\right]$ decreases as $x^{0}$ increases, $\sum_{i} v^{i}>0$. The Kasner exponents $p^{\mu}$, which are normalized to $\sum_{i} p^{i}=1$, are related to the $v^{\mu}$ through $p^{\mu}=v^{\mu} /\left(\sum_{i} v^{i}\right)$.

Consider the trajectories that get inside the future light cone of some point, say the origin ${ }^{4}$. One can radially project these trajectories on the hyperbolic space $H_{d}$ (if a dilaton is present) or $H_{d-1}$ (no dilaton), realized as the future sheet of the unit hyperboloid of vectors with norm squared equal to -1 . This is most readily done by introducing hyperbolic coordinates,

$$
\beta^{\mu}=\rho \gamma^{\mu},
$$

where $\gamma^{\mu}$ are coordinates on the future sheet of the unit hyperboloid,

$$
\gamma^{\mu} \gamma_{\mu}=-1
$$

${ }^{3}$ The Kasner metric (2.1) corresponds to the particular choice of integration constants $\sum_{i} v^{i}=1, \beta_{0}^{\mu}=0$, which implies $t=\exp \left(-x^{0}\right)$, i.e., $x^{0}=\tau$ and $\sqrt{g}=t$. These "initial conditions" can be reached by using the symmetries of the problem. These are (i) independent rescalings of the coordinates $x^{i} \rightarrow x^{i}=k^{i} x^{i}$; (ii) redefinition of the time $x^{0}, x^{0} \rightarrow x^{0}=B x^{0}+C$, where $B$ and $C$ are constants, with $B=k^{1} k^{2} \cdots k^{d}$ so as to preserve the gauge condition $d t=-\sqrt{g} d x^{0}$; and (iii) $\phi \rightarrow \phi^{\prime}=\phi+A$, where $A$ is a constant. However, if one considers many Kasner solutions simultaneously, as we shall do below when analysing collisions from one Kasner regime to another one, one cannot make these simple choices of integration constants for all the solutions at the same time - one can make it only, say, for the "first" one. For this reason, we shall keep generic $\beta_{0}^{\mu}$ in (2.17) and we shall not impose $\sum_{i} v^{i}=1$.

${ }^{4}$ This occurs for $x^{0}$ big enough when $v_{\mu} \beta_{0}^{\mu}<0$ since $\beta^{\mu} \beta_{\mu}=2 v_{\mu} \beta_{0}^{\mu} x^{0}+\beta_{0 \mu} \beta_{0}^{\mu}$. The condition $v_{\mu} \beta_{0}^{\mu}<0$ can always be assumed to hold by shifting the origin of the $\beta^{i}$ 's if necessary. 
In terms of $\rho$ and $\gamma^{\mu}$, the metric becomes

$$
d \sigma^{2}=-d \rho^{2}+\rho^{2} d \Sigma^{2}
$$

where $d \Sigma^{2}$ is the metric on the unit hyperboloid. Reinstating the rescaled lapse $\tilde{N}$, the Hamiltonian is

$$
H=\frac{\tilde{N}}{4}\left[-\pi_{\rho}^{2}+\frac{1}{\rho^{2}} \pi_{\gamma}^{2}\right]
$$

where $\pi_{\gamma}$ are the momenta conjugate to the (constrained) $\gamma^{\mu}$. Equivalently,

$$
H=\frac{\tilde{N}}{4 \rho^{2}}\left[-\pi_{T}^{2}+\pi_{\gamma}^{2}\right]
$$

with $T=\ln \rho$ and $\pi_{T}$ its conjugate momentum. In terms of these variables, the motion is simple in the gauge $\tilde{N}=\rho^{2}$, since the Hamiltonian becomes then just $(1 / 4)\left[-\left(\pi_{T}\right)^{2}+\left(\pi_{\gamma}\right)^{2}\right]$. One can view $T$ as an intrinsic time variable in terms of which one can describe the dynamics. The evolution is a geodesic motion on $\Gamma$.

On the solutions, $x^{0}$ (in the gauge $\tilde{N}=1$ ) $\sim \ln \sqrt{g}, \rho \sim|\ln \sqrt{g}|^{1 / 2}$ and $T \sim \ln |\ln \sqrt{g}| \sim \ln |\ln t|\left(v_{\mu} \beta_{0}^{\mu}<0\right)$. Small volume is thus equivalent to large $\rho$ or what is the same, large $T$, i.e., large intrinsic time. We shall call the limit $\rho \rightarrow \infty$ the BKL limit.

\section{Kasner solution - Non-diagonal case}

\subsection{Iwasawa decomposition}

In the homogeneous, vacuum context, the metric remains diagonal if the initial data are so. This is in general not true when matter or inhomogeneities are included, in which case off-diagonal components generically appear even if there is none initially. For this reason, it is important to understand the rôle of off-diagonal terms already in the simpler homogeneous context, by examining the evolution of initial data that are not diagonal. This is most simply done by performing a constant linear transformation on the Kasner solution, which is a symmetry for Bianchi I models. If $L$ is the linear transformation needed to diagonalize the initial data, it is easy to see that the solution is given by

$$
G(t)={ }^{t} L G_{K}(t) L
$$


where ${ }^{t}$ denotes transposition and $G_{K}(t)$ is the above diagonal Kasner solution. The dilaton, being a scalar, is still given by the same expression. Note that $\operatorname{det} G(t)=(\operatorname{det} L)^{2} t^{2}$.

To understand the qualitative behaviour of (3.1), it is convenient to perform the Iwasawa decomposition of the metric,

$$
G={ }^{t} \mathcal{N} \mathcal{A}^{2} \mathcal{N}
$$

where $\mathcal{N}$ is an upper triangular matrix with 1's on the diagonal $\left(\mathcal{N}_{i i}=1\right.$, $\mathcal{N}_{i j}=0$ for $i>j$ ) and where $\mathcal{A}$ is a diagonal matrix with positive elements, which we parametrize as

$$
\mathcal{A}=\exp (-\beta), \beta=\operatorname{diag}\left(\beta^{1}, \beta^{2}, \cdots, \beta^{d}\right) .
$$

One can view the Iwasawa decomposition ${ }^{5}$ as the Gram-Schmidt orthogonalisation of the initial basis, which is indeed a triangular process,

$$
d s^{2}=g_{i j} d x^{i} d x^{j}=\sum_{k=1}^{d} e^{\left(-2 \beta_{k}\right)}\left(\theta^{k}\right)^{2}
$$

with

$$
\theta^{k}=\sum_{i} \mathcal{N}_{k i} d x^{i}
$$

Starting with $\theta^{d}=d x^{d}$, one successively constructs the next $\theta^{j}$ 's by adding linear combinations of the $d x^{p}$ 's $(p>j)$ in such a way that $\theta^{d-1}$ is orthogonal to $\theta^{d}, \theta^{d-2}$ is orthogonal to both $\theta^{d}$ and $\theta^{d-1}$, etc. Explicitly,

$$
\begin{aligned}
\theta^{d} & =d x^{d} \\
\theta^{d-1} & =d x^{d-1}+\mathcal{N}_{d-1 d} d x^{d} \\
\theta^{d-2} & =d x^{d-2}+\mathcal{N}_{d-2 d-1} d x^{d-1}+\mathcal{N}_{d-2 d} d x^{d} \\
& \cdots
\end{aligned}
$$

Note that the dual basis $\left\{e_{j}\right\}$ reads

$$
e_{j}=\sum_{m}\left(\mathcal{N}^{-1}\right)_{m j} \frac{\partial}{\partial x^{m}} .
$$

\footnotetext{
${ }^{5}$ The Iwasawa decomposition applies to general symmetric spaces (see e.g. [H78]). In our case the relevant symmetric space is the coset space $S L(d, \mathbb{R}) / S O(d)$ since the space of positive definite symmetric matrices can be identified with $G L(d, \mathbb{R}) / O(d)$, which is isomorphic to $S L(d, \mathbb{R}) / S O(d) \times \mathbb{R}^{+}$.
} 
The variables $\beta^{i}$ of the Iwasawa decomposition give the scale factors in the new, orthogonal, basis, while the $\mathcal{N}_{i j}$ characterize the change of basis that diagonalizes the metric and hence, parametrize the off-diagonal components of the original $g_{i j}$.

In the diagonal case, $\mathcal{N}=\mathbf{1}$ and the $\beta$ 's behave linearly with $\tau$,

$$
\beta^{i}=p^{i} \tau \quad \text { (diagonal Kasner). }
$$

In the general case, $\mathcal{N} \neq \mathbf{1}$ and the evolution of the variables $\beta^{i}, \mathcal{N}_{i j}$ of the Iwasawa decomposition is more complicated. However, it becomes simple for $\tau \rightarrow \pm \infty$. Indeed, the $\beta$ 's become asymptotically linear functions of $\tau$, as in the diagonal case, with coefficients that are given by a permutation of the $p^{i}$ 's, whereas the $\mathcal{N}_{i j}$ become constant. More precisely, let $q^{1}, q^{2}, \cdots, q^{d}$ be the ordered Kasner exponents, $q^{1} \leq q^{2} \leq \cdots \leq q^{d}, q^{i}=p^{\sigma(i)}$, with $\sigma$ an appropriate permutation of $1,2, \cdots, n$. Then one finds (for generic $L$ 's):

- as $\tau \rightarrow-\infty(t \rightarrow \infty), \beta^{i} \sim q^{d-i} \tau$ and $\mathcal{N}_{i j} \rightarrow$ const;

- as $\tau \rightarrow+\infty\left(t \rightarrow 0^{+}\right), \beta^{i} \sim q^{i} \tau$ and $\mathcal{N}_{i j} \rightarrow$ const.

In both limits, one has $\beta^{1} \leq \beta^{2} \leq \cdots \leq \beta^{d}$. The motion does not change the Kasner exponents $p^{i}$, but just redistributes them among the $\beta$ 's so as to achieve $\beta^{1} \leq \beta^{2} \leq \cdots \leq \beta^{d}$ for both $\tau \rightarrow \infty$ and $\tau \rightarrow-\infty$. [The fact that one gets $\beta^{1} \leq \beta^{2} \leq \cdots \leq \beta^{d}$ (in that order) follows from the choice for the matrix $\mathcal{N}$ made in the Iwasawa decomposition; had we taken $\mathcal{N}$ to be e.g. lower triangular instead of upper-triangular, the inequalities would have been all reversed.]

One can arrive at the above conclusions concerning the asymptotic form of the solutions either by direct computation from (3.1) and (3.2), or by using the Hamiltonian formulation of the theory. We shall derive here the Hamiltonian analysis and give the direct calculation (in the specific $3+1$ case) in the first appendix.

\subsection{Symmetry walls}

The simplest way to understand the asymptotic dynamics of the scale factors in the non-diagonal case is to use the geometric picture developed in subsection 2.2. 


\subsubsection{BKL limit}

Using the Iwasawa decomposition, the supermetric (2.11) becomes

$$
\begin{gathered}
d \sigma^{2}=\operatorname{tr}(d \beta)^{2}-(\operatorname{tr} d \beta)^{2}+\frac{1}{2} \operatorname{tr}\left[\mathcal{A}^{2} d \mathcal{N} \mathcal{N}^{-1} \mathcal{A}^{-2}{ }^{t}\left(\mathcal{N}^{-1}\right) d\left({ }^{t} \mathcal{N}\right)\right] \\
+(d \phi)^{2}
\end{gathered}
$$

i.e.,

$$
\begin{aligned}
d \sigma^{2}= & \sum_{i=1}^{d}\left(d \beta^{i}\right)^{2}-\left(\sum_{i=1}^{d} d \beta^{i}\right)^{2} \\
& +\frac{1}{2} \sum_{i<j} e^{2\left(\beta^{j}-\beta^{i}\right)}\left(\left(d \mathcal{N} \mathcal{N}^{-1}\right)_{i j}\right)^{2}+d \phi^{2}
\end{aligned}
$$

For $d=3$, this expression reduces to the one explicitly given in [HPT82].

The Hamiltonian governing the evolution is therefore

$$
\begin{array}{r}
H=\frac{\tilde{N}}{4}\left[\sum_{i=1}^{d}\left(\pi_{i}\right)^{2}-\frac{1}{d-1}\left(\sum_{i=1}^{d} \pi_{i}\right)^{2}+\left(\pi_{\phi}\right)^{2}\right] \\
+\frac{\tilde{N}}{2} \sum_{i<j} e^{-2\left(\beta^{j}-\beta^{i}\right)}\left(\sum_{m} P_{i m} \mathcal{N}_{j m}\right)^{2}
\end{array}
$$

where the $P_{i m}$ are the momenta conjugate to the $\mathcal{N}_{i m}, P_{i m}=\partial \mathcal{L} / \partial \dot{\mathcal{N}}_{i m}$. Because the terms involving the off-diagonal parameters are positive, the constraint $H=0$ implies that the velocity $\dot{\beta}^{\mu}$ is timelike or null.

We are interested in the BKL limit, i.e. small volume (large $\sum_{i} \beta^{i}$ ) or large $\rho$. Making the change of variables (2.19) yields, instead of (2.23)

$$
H=\frac{\tilde{N}}{\rho^{2}}\left[\frac{1}{4}\left(-\pi_{T}^{2}+\pi_{\gamma}^{2}\right)+\frac{\rho^{2}}{2} \sum_{i<j} e^{-2 \rho\left(\gamma^{j}-\gamma^{i}\right)}\left(\sum_{m} P_{i m} \mathcal{N}_{j m}\right)^{2}\right]
$$

As $\rho$ becomes large, the terms $\rho^{2} \exp \left[-2 \rho\left(\gamma^{j}-\gamma^{i}\right)\right]$ either go to zero if $\gamma^{j}-\gamma^{i}$ is positive, or explode to $+\infty$ if $\gamma^{j}-\gamma^{i}$ is negative. Thus one can replace the exponentials by $\Theta\left[-2\left(\gamma^{j}-\gamma^{i}\right)\right]$, where $\Theta(x)=0$ for $x<0$ and $+\infty$ for $x>0^{6}$. Of course, $\Theta(2 x)=\Theta(x)$, but we keep the factor 2 here to recall that

\footnotetext{
${ }^{6}$ One should more properly write $\Theta_{\infty}(x)$, but since this is the only step function encountered in this article, we use the simpler notation $\Theta(x)$.
} 
the arguments of the exponentials from which the $\Theta$-functions originate come with a well-defined normalization. Since the functions $\gamma^{j}-\gamma^{i}$ and $\beta^{j}-\beta^{i}$ have the same sign $(\rho>0)$ and since $\Theta(x)=\lambda \Theta(x)$ for $\lambda>0$, one can replace $\Theta\left[-2\left(\gamma^{j}-\gamma^{i}\right)\right]$ by $\Theta\left[-2\left(\beta^{j}-\beta^{i}\right)\right]$. The Hamiltonian governing the dynamics of the scale factors $\beta^{\mu}$ for type-I, non-diagonal models, reads, in the BKL limit,

$$
\begin{gathered}
H=\frac{\tilde{N}}{4}\left[\sum_{i=1}^{d} \pi_{i}^{2}-\frac{1}{d-1}\left(\sum_{i=1}^{d} \pi_{i}\right)^{2}+\pi_{\phi}^{2}\right. \\
\left.+\sum_{i<j} \Theta\left[-2\left(\beta^{j}-\beta^{i}\right)\right]\right]
\end{gathered}
$$

The terms $\Theta\left[-2\left(\beta^{j}-\beta^{i}\right)\right]$ are present for all $(i, j)$ with $i<j$ provided all non-negative terms $\left(\sum_{m} P_{i m} \mathcal{N}_{j m}\right)^{2}$ are different from zero. This is the case generically, i.e., for all initial data except a set of measure zero. For this reason, we shall assume that it is fulfilled from now on. As a rule, we shall always make similar "genericity" assumptions below concerning the coefficients of the various potentials that enter the discussion.

\subsubsection{First encounter with billiards}

Because of the simplicity of (3.16), the dynamics is easy to describe in the BKL limit. First, since the off-diagonal parameters $\mathcal{N}_{i j}$ and their conjugate drop out from the Hamiltonian, they are constant for large $\rho$. This is what we saw in subsection (3.1). The only effect of the off-diagonal components is to induce the $\Theta$-function potentials for the scale factors. The asymptotic freezing of the off-diagonal terms is further discussed in appendix B. Second, the $\Theta$-terms constrain the scale factors to be in the region

$$
w_{(i j)}^{S}(\beta) \equiv w_{(i j) \mu}^{S} \beta^{\mu} \equiv \beta^{j}-\beta^{i} \geq 0 \quad(i<j) .
$$

In that region the motion is free, i.e., is given by a lightlike straight line (2.17) for some $v^{\mu}$ fulfilling (2.18), exactly as for diagonal Kasner metrics. When the trajectory hits a wall $\beta^{j}-\beta^{i}=0$, it undergoes a reflection. This reflection permutes two $v^{\mu}$ 's (see below). After a finite number of collisions, the $v^{\mu}$ 's get ordered, $v^{1} \leq v^{2} \cdots \leq v^{d}$, and the motion then goes on freely forever. Indeed, the subsequent evolution of the $\beta$ 's, once the $v^{\mu}$ 's are ordered, can never hit a wall (where two $\beta$ 's are equal) any more. This is what we announced in 
the analysis of the asymptotic behaviour of the solutions in subsection 3.1. We stress that this simple evolution arises only in the BKL limit. Before the asymptotic regime is reached, one cannot replace the exponentials by $\Theta$-functions. The collisions that take place then are not strictly localized in time and therefore may not be clearly separated from each other. In addition, there is a slow drift of the off-diagonal parameters.

The region $w_{(i j)}^{S}(\beta) \geq 0(i<j), \sum_{i} \beta^{i} \geq 0$ is a convex region of the space of the scale factors. We shall call the hyperplanes $w_{(i j)}^{S}(\beta)=0$ the "symmetry walls". These hyperplanes are timelike since

$$
G^{\mu \nu} w_{(i j) \mu}^{S} w_{(i j) \nu}^{S}=2
$$

and intersect therefore the hyperboloid $G_{\mu \nu} \beta^{\mu} \beta^{\nu}=-1, \sum_{i} \beta^{i} \geq 0$. The symmetry billiard is defined to be the region of hyperbolic space determined by these inequalities.

Among the $d(d-1) / 2$ symmetry walls, only $d-1$ are relevant. Indeed, the inequalities (3.17) all follow from $w_{(i i+1)}^{S} \geq 0$. The symmetry billiard is thus defined by the $d-1$ inequalities

$$
\beta^{2}-\beta^{1} \geq 0, \beta^{3}-\beta^{2} \geq 0, \cdots, \beta^{d}-\beta^{d-1} \geq 0
$$

and extend to infinity. It has infinite volume.

In order to analyse the collision against the wall $\beta^{i+1}-\beta^{i}=0$, we make linear redefinitions so that $\beta^{i+1}-\beta^{i}$ is one of the new variables,

$$
\beta^{\mu} \rightarrow \beta^{\mu}(\mu \neq i, i+1), u=\beta^{i+1}+\beta^{i}, z=\beta^{i+1}-\beta^{i}
$$

In these new variables, the potential associated with the symmetry wall $\beta^{i+1}-$ $\beta^{i}=0$ is simply $\Theta(-2 z)$. Furthermore, since $z$ is orthogonal to the other variables, the sole effect of the collision is to change the sign of $\dot{z}$, leaving the other velocities fixed. In terms of the original variables, this is just the permutation of the velocities $v^{i}$ and $v^{i+1}$,

$$
v^{i} \rightarrow v^{i+1}, \quad v^{i+1} \rightarrow v^{i} \quad \text { (other } v^{k} \text { unchanged) }
$$

This transformation belongs to the orthochronous Lorentz group because it preserves both the Lorentzian metric and the arrow of time $\left(G_{\mu \nu} v^{\prime \mu} v^{\prime \nu}=\right.$ $\left.G_{\mu \nu} v^{\mu} v^{\nu}, \sum_{i} v^{i}=\sum_{i} v^{i}\right)$. More generally, an arbitrary number of collisions against the symmetry walls will lead to an arbitrary permutation of the 
$v^{i}$ 's, since the transpositions $(i, i+1)$ generate the symmetry group $S_{d}$ in $d$ elements.

\section{Remarks}

\section{Large volume limit}

It follows from the above analysis that in the small volume limit, the Kasner exponents get re-ordered so that the first spatial direction carries the smallest Kasner exponent, the second spatial direction carries the next to smallest Kasner exponent etc. In the particular homogeneous case considered here, one can also investigate the large volume limit by the same techniques. Although this cannot be extended to the general inhomogeneous case discussed below, we briefly indicate how this is achieved in the present special case.

In the large volume limit, the $\beta$ 's go to the past in the space of the scale factors. So one sets

$$
\beta^{\mu}=-\rho \gamma^{\mu},
$$

where $\gamma^{\mu}$ are still coordinates on the future sheet of the unit hyperboloid, $\gamma^{\mu} \gamma_{\mu}=-1$. Making the same transformations as above, one finds that all the symmetry walls come with the other sign, i.e., in the large volume limit, the potential reduces to $\sum_{i>j} \Theta\left[-2\left(\beta^{j}-\beta^{i}\right]\right.$. This

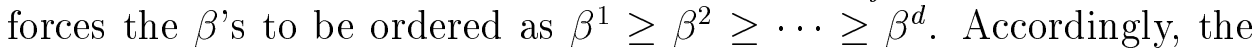
collisions against the walls reorders now the velocities $p^{i}$ in decreasing order, as we stated in subsection 3.1.

\section{Are the symmetry walls a gauge artifact?}

Since the metric can diagonalized at all times by a time-independent coordinate transformation $x^{a} \rightarrow x^{a}=L^{a}{ }_{b} x^{b}$, one might fear that the symmetry walls, which are related to the off-diagonal components, are somewhat a gauge artifact with no true physical content. This conclusion, however, would be incorrect. First, the transformation needed to diagonalize the metric may not be a globally well-defined coordinate transformation if the spatial sections have non-trivial topology, e.g., are tori, since it would conflict in general with periodicity conditions. Second, even if the spatial sections are homeomorphic to $R^{d}$, the transformation $x^{a} \rightarrow x^{a}=L^{a}{ }_{b} x^{b}$, although a diffeomorphism, is 
not a proper gauge transformation in the sense that it is generated by a non-vanishing charge. One should regard as distinct (although related by a symmetry) two solutions that differ by the transformation $x^{a} \rightarrow x^{a}=L^{a}{ }_{b} x^{b}$. Initial conditions for which the metric is diagonal and hence the symmetry walls absent - form a set of measure zero.

\section{Alternative description of symmetry walls}

We have just shown that the Iwasawa decomposition of the spatial metric leads to a projected description of the $G L(d, \mathbb{R}) / O(d)$-geodesics as motions in the space of the scale factors with exponential ("Todalike") potentials. An alternative description exists, which is based on the decomposition $G={ }^{t} R A R$ of the spatial metric, where $R \in$ $S O(d)$ and $A$ is diagonal [R72a, KM02]. One then gets "Calogero-like" $\sinh ^{-2}\left(\beta^{i}-\beta^{j}\right)$-potentials. In the BKL limit, these potentials can be replaced by sharp wall potentials but whether the system lies to the left or to the right of the wall $\beta^{i}-\beta^{j}=0$ depends in this alternative description on the initial conditions.

\section{Asymptotic dynamics in the general case - Gravitational billiards}

We shall now show that the same ideas apply in the general case described by the action (1.1). In the vicinity of a spacelike singularity, the spatial points decouple and the billiard picture remains valid at each spatial point. Inhomogeneities and $p$-forms bring in only new walls.

\subsection{Pseudo-Gaussian coordinates}

We thus assume that there is a spacelike singularity at a finite distance in proper time. We adopt a spacetime slicing adapted to the singularity, which "occurs" on a slice of constant time. We build the slicing from the singularity by taking pseudo-Gaussian coordinates defined by $\tilde{N}=1$ and $N^{i}=0$, where $\tilde{N}$ is again the rescaled lapse $\tilde{N}=N / \sqrt{g}$ and $N^{i}$ is the standard shift. More precisely, we assume that in some spacetime patch, the metric reads

$$
d s^{2}=-g\left(x^{0}, x^{i}\right)\left(d x^{0}\right)^{2}+g_{i j}\left(x^{0}, x^{i}\right) d x^{i} d x^{j}, \quad g \equiv \operatorname{det}\left(g_{i j}\right),
$$


where the local volume $g$ collapses at each spatial point as $x^{0} \rightarrow+\infty$, in such a way that the proper time $d t=-\sqrt{g} d x^{0}$ remains finite (and tends conventionally to $0^{+}$). We shall make the further assumption that since the local volume tends to zero, the variable $\rho$ introduced above can be used everywhere in a given region of space (in a neighborhood of the singularity) as a well-defined ("intrinsic") time variable that goes all the way to $+\infty$. We can then investigate the BKL limit $\rho \rightarrow \infty$. Differently put, we study the general solution of the equations of motion in the regime met above where the scale factors $\beta^{\mu}$ go to infinity inside the future light cone in superspace, $\beta^{\mu}=\rho \gamma^{\mu}, G_{\mu \nu} \gamma^{\mu} \gamma^{\nu}=-1, \sum_{i} \gamma^{i}>0, \rho \rightarrow \infty$ (at each spatial point in the local region under consideration). Some aspects of the consistency of this limit are discussed in the appendix D.

One of the motivations for studying spacelike singularities comes from the singularity theorems [HP70]. Of course, not all singularities are spacelike, and furthermore, there is no claim here that all spacelike singularities are necessarily of the BKL type. We are just interested in this regime, which, as we shall see, has a high degree of self-consistency and is quite general in the sense that it involves as many arbitrary functions of space as the general solution does (see subsection 4.6).

The only coordinate freedom left in the pseudo-Gaussian gauge (after having fixed the hypersurface $t=0$ ) is that of making time-independent changes of spatial coordinates $x^{k} \rightarrow x^{\prime k}=f^{k}\left(x^{m}\right)$. Since the rescaled lapse is a spatial density of weight minus one, such changes of coordinates have the unusual feature of also changing the slicing (in some determined way - the scalar lapse $N$ changes in a well-defined manner).

The fields $\phi$ and $A_{\mu_{1} \cdots \mu_{p}}^{(p)}$ are also a priori functions of both space and time. No symmetry condition is imposed. Although the equations at each point will be asymptotically the same as those of homogeneous cosmological models, this does not follow from imposing extra dimensional reduction conditions but is rather a direct consequence of the general dynamical equations.

We shall partially fix the gauge in the $p$-form sector by imposing the temporal gauge condition $A_{0 \mu_{2} \cdots \mu_{p}}^{(p)}=0$. This leaves the freedom of performing time-independent gauge transformations.

\subsection{Hamiltonian action}

To focus on the features relevant to the billiard picture, we assume first that there is no Chern-Simons terms or couplings of the exterior form gauge 
fields through a modification of the curvatures $F^{(p)}$, which are thus taken to be Abelian, $F^{(p)}=d A^{(p)}$. We verify in subsection 4.7 below that these interaction terms do not change the analysis. The Hamiltonian action in the pseudo-Gaussian gauge reads

$$
\begin{aligned}
& S\left[g_{i j}, \pi^{i j}, \phi, \pi_{\phi}, A_{m_{1} \cdots m_{p}}^{(p)}, \pi_{(p)}^{m_{1} \cdots m_{p}}\right]= \\
& \quad \int d x^{0}\left(\int d^{d} x\left(\pi^{i j} \dot{g}_{i j}+\pi_{\phi} \dot{\phi}+\sum_{p} \pi_{(p)}^{m_{1} \cdots m_{p}} \dot{A}_{m_{1} \cdots m_{p}}^{(p)}\right)-H\right)
\end{aligned}
$$

where the Hamiltonian is

$$
\begin{aligned}
H & =\int d^{d} x \mathcal{H} \\
\mathcal{H} & =L+M \\
L & =\pi^{i j} \pi_{i j}-\frac{1}{d-1}\left(\pi_{i}^{i}\right)^{2}+\frac{1}{4}\left(\pi_{\phi}\right)^{2}+ \\
& \sum_{p} \frac{p ! e^{-\lambda^{(p)} \phi}}{2} \pi_{(p)}^{m_{1} \cdots m_{p}} \pi_{(p) m_{1} \cdots m_{p}} \\
M= & -R g+g^{i j} g \partial_{i} \phi \partial_{j} \phi+\sum_{p} \frac{e^{\lambda^{(p)} \phi}}{2(p+1) !} g F_{m_{1} \cdots m_{p+1}}^{(p)} F^{(p) m_{1} \cdots m_{p+1}}
\end{aligned}
$$

The dynamical equations of motion are obtained by varying the above action w.r.t. the spatial metric components, the dilaton, the spatial $p$-form components and their conjugate momenta. In addition, there are constraints on the dynamical variables, which are

$$
\begin{array}{rll}
\mathcal{H} & =0 & \text { ("Hamiltonian constraint") } \\
\mathcal{H}_{i} & =0 \quad \text { ("momentum constraint" }), \\
\varphi_{(p)}^{m_{1} \cdots m_{p-1}} & =0 \quad \text { ("Gauss law" for each } p \text {-form })(p>0)
\end{array}
$$

Here, we have set

$$
\begin{aligned}
\mathcal{H}_{i} & =-2 \pi_{i \mid j}^{j}+\pi_{\phi} \partial_{i} \phi+\sum_{p} \pi_{(p)}^{m_{1} \cdots m_{p}} F_{i m_{1} \cdots m_{p}}^{(p)} \\
\varphi_{(p)}^{m_{1} \cdots m_{p-1}} & =-p \pi_{(p)}^{m_{1} \cdots m_{p-1} m_{p}}{ }_{\mid m_{p}}
\end{aligned}
$$

In order to understand the asymptotic behaviour of the fields, we perform, at each spatial point, the Iwasawa decomposition of the metric. This is a 
point canonical transformation, extended to the momenta in the standard way, i.e., $\pi^{i j} \dot{g}_{i j}=\pi_{i} \dot{\beta}^{i}+\sum_{i<j} P_{i j} \dot{\mathcal{N}}_{i j}$. We then split the Hamiltonian in two parts, one denoted by $K$, which is the kinetic term for the local scale factors $\beta^{\mu}$, and the other denoted by $V$, which contains everything else. It is indeed natural to group the kinetic term for the off-diagonal variables with the original potential terms because they asymptotically play the role of a potential for the scale factors. As we shall see, the same feature holds for the $p$-form kinetic terms. In fact, this is not surprising, at least when $p=1$, since 1-forms can be viewed as off-diagonal components of the metric in one dimension higher. Thus, we write

$$
\begin{aligned}
\mathcal{H} & =K+V \\
K & =\frac{1}{4}\left[\sum_{i=1}^{d} \pi_{i}^{2}-\frac{1}{d-1}\left(\sum_{i=1}^{d} \pi_{i}\right)^{2}+\pi_{\phi}^{2}\right] \\
V & =V_{S}+V_{G}+\sum_{p} V_{p}+V_{\phi} \\
V_{S} & =\frac{1}{2} \sum_{i<j} e^{-2\left(\beta^{j}-\beta^{i}\right)}\left(\sum_{m} P_{i m} \mathcal{N}_{j m}\right)^{2} \\
V_{G} & =-R g \\
V_{(p)} & =V_{(p)}^{e l}+V_{(p)}^{\text {magn }} \\
V_{(p)}^{e l} & =\frac{p ! e^{-\lambda^{(p)} \phi}}{2} \pi_{(p)}^{m_{1} \cdots m_{p}} \pi_{(p) m_{1} \cdots m_{p}} \\
V_{(p)}^{\text {magn }} & =\frac{e^{\lambda^{(p)} \phi}}{2(p+1) !} g F_{m_{1} \cdots m_{p+1}}^{(p)} F^{(p) m_{1} \cdots m_{p+1}} \\
V_{\phi} & =g^{i j} g \partial_{i} \phi \partial_{j} \phi .
\end{aligned}
$$

We know that in the BKL limit, the symmetry potential $V_{S}$ becomes a sum of sharp wall potentials,

$$
V_{S} \simeq \sum_{i<j} \Theta\left[-2\left(\beta^{j}-\beta^{j}\right)\right] \quad(\rho \rightarrow \infty)
$$

The computation that leads to (4.21) was carried out above in the spatially homogeneous context but remains clearly valid here since $V_{S}$ contains no spatial gradients. This potential is "ultralocal", i.e., its value at any spatial point involves only the scale factors at that same spatial point. It is quite 
remarkable that the same sharp wall behavior emerges asymptotically for the other potentials.

\subsection{Curvature (gravitational) walls}

We first establish this fact for the gravitational potential.

\subsubsection{Computation of curvature}

To that end, one must explicitly express the spatial curvature in terms of the scale factors and the off-diagonal variables $\mathcal{N}_{i j}$. The calculation is most easily done in the frame $\theta^{k}=\sum_{i} \mathcal{N}_{k i} d x^{i}$ in which the spatial metric is diagonal,

$$
d s^{2}=\sum_{k=1}^{d} a_{k}^{2}\left(\theta^{k}\right)^{2}, \quad a_{k}=e^{-\beta^{k}}
$$

Let $C^{i}{ }_{m n}(x)$ be the structure functions of the basis $\left\{\theta^{k}\right\}$,

$$
d \theta^{i}=-\frac{1}{2} C_{m n}^{i} \theta^{m} \theta^{n}
$$

Here, $d$ is the spatial exterior differential. The functions $C^{i}{ }_{m n}(x)$ depend clearly only on the off-diagonal variables $\mathcal{N}_{i j}$ and not on the scale factors. Using the Cartan formulas for the connection 1-form $\omega^{i}{ }_{j}$,

$$
\begin{aligned}
& d \theta^{i}+\sum_{j} \omega^{i}{ }_{j} \wedge \theta^{j}=0 \\
& d \gamma_{i j}=\omega_{i j}+\omega_{j i}
\end{aligned}
$$

where $\gamma_{i j}=\delta_{i j} a_{i}^{2}$ is the metric in the frame $\left\{\theta^{k}\right\}$, one finds

$$
\begin{aligned}
\omega_{\ell}^{k}= & \sum_{j} \frac{1}{2}\left(C^{j}{ }_{k \ell} \frac{a_{j}^{2}}{a_{k}^{2}}+C^{\ell}{ }_{k j} \frac{a_{\ell}^{2}}{a_{k}^{2}}-C_{\ell j}^{k}\right) \theta^{j} \\
& +\sum_{j} \frac{1}{2 a_{k}^{2}}\left(\delta_{k \ell}\left(a_{k}^{2}\right)_{, j}+\delta_{k j}\left(a_{k}^{2}\right)_{, \ell}-\delta_{\ell j}\left(a_{\ell}^{2}\right)_{, k}\right) \theta^{j}
\end{aligned}
$$

One then gets the Riemann tensor $R_{\ell m n}^{k}$, the Ricci tensor $R_{\ell n}$ and the scalar curvature $R$ through

$$
\mathcal{R}_{\ell}^{k}=d \omega_{\ell}^{k}+\sum_{j} \omega^{k}{ }_{j} \omega_{\ell}^{j}
$$




$$
=\frac{1}{2} \sum_{m, n} R_{\ell m n}^{k} \theta^{m} \wedge \theta^{n}
$$

$\left(\mathcal{R}^{k}{ }_{\ell}\right.$ is the curvature 2 -form) and

$$
R_{\ell n}=R_{\ell k n}^{k}, \quad R=\sum_{\ell} \frac{1}{a_{\ell}^{2}} R_{\ell \ell}
$$

Direct, but somewhat cumbersome, computations yield

$$
R=-\frac{1}{4} \sum_{j, k, \ell} \frac{a_{j}^{2}}{a_{\ell}^{2} a_{k}^{2}}\left(C^{j}{ }_{k \ell}\right)^{2}+\sum_{j} \frac{1}{a_{j}^{2}} F_{j}\left(\partial^{2} \beta, \partial \beta, \partial C, C\right)
$$

where $F_{j}$ is some complicated function of its arguments whose explicit form will not be needed here. The only property of $F_{j}$ that will be of importance is that it is a polynomial of degree two in the derivatives $\partial \beta$ and of degree one in $\partial^{2} \beta$. Thus, the exponential dependence on the $\beta^{i}$ s, which determines the asymptotic behaviour in the BKL limit, occurs only through the $a_{j}^{2}$-terms written explicitly in (4.30).

Without loss of generality, we can assume $j \neq k$ and $j \neq \ell$ in the first sum on the right-hand side of (4.30), since the terms with either $j=k$ or $j=\ell$ can be absorbed through a redefinition of $F_{j}$. Also, one has clearly $k \neq \ell$ because the structure functions $C^{j}{ }_{k \ell}$ are antisymmetric in $k, \ell$. We can thus write the gravitational potential as

$$
V_{G} \equiv-g R=\frac{1}{4} \sum_{i \neq j, i \neq k, j \neq k} e^{-2 \alpha_{i j k}(\beta)}\left(C_{j k}^{i}\right)^{2}-\sum_{j} e^{-2 \mu_{j}(\beta)} F_{j}
$$

where the linear forms $\alpha_{i j k}(\beta)$ and $\mu_{j}(\beta)$ are given by

$$
\alpha_{i j k}(\beta)=2 \beta^{i}+\sum_{m,(m \neq i, m \neq j, m \neq k)} \beta^{m}, \quad(i \neq j, i \neq k, j \neq k)
$$

and

$$
\mu_{j}(\beta)=\sum_{m,(m \neq j)} \beta^{m}
$$

respectively. 


\subsubsection{BKL limit}

In the BKL limit where the scale factors $\beta^{\mu}$ go to infinity inside the future light cone, the gravitational potential becomes a sum of sharp wall potentials,

$$
V_{G} \simeq \sum_{i \neq j, i \neq k, j \neq k} \Theta\left[-2 \alpha_{i j k}(\beta)\right]+\sum_{i}\left( \pm \Theta\left[-2 \mu_{i}(\beta)\right]\right)
$$

The terms in the second sum seem to pose a problem because they do not have a definite sign. They are, however, in fact always zero in the BKL limit because $\mu_{i}(\beta)>0$. Indeed, each linear form $\mu_{i}(\beta)$ is lightlike and hence, each hyperplane $\mu_{i}(\beta)=0$ is tangent to the light cone along some generatrix. This means that the future light cone is entirely on one side of the hyperplane $\mu_{i}(\beta)=0$ (i.e., either $\mu_{i}(\beta)>0$ for all points inside the future light cone or $\left.\mu_{i}(\beta)<0\right)$. Now, the point $\beta^{1}=\beta^{2}=\cdots=\beta^{d}=1$ is inside the future light cone and makes all the $\mu_{i}$ 's positive. Hence $\mu_{i}(\beta)>0$ inside the future light cone for each $i$ and $\Theta\left[-2 \mu_{i}(\beta)\right]=0$ : we can drop the second term in the gravitational potential, which reduces to

$$
V_{G} \simeq \sum_{i \neq j, i \neq k, j \neq k} \Theta\left[-2 \alpha_{i j k}(\beta)\right]
$$

Note that in $D=3$ spacetime dimensions, the gravitational walls $\alpha_{i j k}(\beta)=0$ $(i \neq j, i \neq k, j \neq k)$ are absent, since one cannot find three distinct spatial indices. The first term in (4.30) is of the same type as the second term: the only gravitational walls are then all of the subdominant type $\mu_{j}$ and thus, in the BKL limit,

$$
V_{G} \simeq 0 \quad(D=3)
$$

We thus see that the gravitational potential becomes, in the BKL limit, a positive sum of sharp wall potentials,

$$
V_{G} \simeq \sum_{i \neq j, i \neq k, j \neq k} \Theta\left[-2 \alpha_{i j k}(\beta)\right](D>3), \quad V_{G} \simeq 0 \quad(D=3)
$$

This is remarkable for many reasons. First, the final form of the potential is rather simple, even though the curvature is a rather complicated function of the metric and its derivatives. Second, the limiting expression of the potential is positive, even though there are (subdominant) terms in $V_{G}$ with indefinite sign. Third, it is ultralocal in the scale factors, i.e. involves only 
the scale factors but not their derivatives. It is this fact that accounts for the decoupling of the various spatial points.

It follows from this analysis that the scale factors are constrained by the conditions

$$
\alpha_{i j k}(\beta) \geq 0 \quad(D>3)
$$

besides the symmetry inequalities (3.19). The hyperplanes $\alpha_{i j k}(\beta)=0$ are called the "curvature" or "gravitational" walls.

\subsubsection{Remarks}

1. In $D>3$ dimensions, one can argue more directly that the first term in $V_{G}$ asymptotically dominates the second one in the BKL limit $\rho \rightarrow \infty$ by observing that the $\mu^{j}$ 's are all positive when the $\alpha_{i j k}$ are positive, since they can be written as linear combinations with positive coefficients of the $\alpha_{i j k}$ (the converse is not true, one may have $\mu^{j}>0$ for all $j$ 's together with $\alpha_{i j k}<0$ for some $\alpha_{i j k}$ 's $)^{7}$. Thus, in the region $\alpha_{i j k}>0$, the exponentials in $V_{G}$ all go to zero as $\rho \rightarrow \infty$ and one can replace $V_{G}$ by zero. Conversely, when one of the $\mu_{j}$ 's is negative, there is always at least one $\alpha_{i j k}$ that is also negative. Actually, assuming without loss of generality that the $\beta^{i}$ 's are ordered, $\beta^{1} \leq \beta^{2} \leq \cdots \leq \beta^{d}$, the most negative $\mu_{i}$ is $\mu_{d}=\beta^{1}+\beta^{2}+\cdots+\beta^{d-1}$. But one has $\alpha_{1 d d-1}=2 \beta^{1}+\beta^{2}+\cdots+\beta^{d-2} \leq \mu^{d}$, in fact $\alpha_{1 d d-1} \leq \alpha_{i j k}$ (for all $i, j, k$ ) and $\alpha_{1 d d-1} \leq \mu_{j}$ (for all $j$ 's). Thus, as $\rho \rightarrow \infty$ (keeping the $\gamma^{\mu}$ fixed), the behaviour of $V_{G}$ is controlled by the exponential $\exp \left(-2 \alpha_{1 d d-1}\right)$, which blows up the fastest. Since it is multiplied by a positive coefficient, we conclude that $V_{G} \rightarrow+\infty$ (even though the subdominant term with $F_{j}$ has no definite $\operatorname{sign}^{8}$ ). If the $\beta^{i}$ were not ordered, it would be a different $\alpha_{i j k}$ that would take over, leading to the same conclusion.

\footnotetext{
${ }^{7}$ In $D=3$, there is no $\alpha_{i j k}$, so this property does not hold. However, when there are $p$-forms present, one can develop the same more direct alternative proof that the $\mu^{j}$ 's can be dropped because one can then express the $\mu^{j}$ as positive linear combinations of the $p$-form wall forms.

${ }^{8}$ Note two things: (i) the exponent $\mu_{d}$ is of the same order as $\alpha_{1 d d-1}$ when $\beta^{1} \simeq$ $\beta^{2} \simeq \beta^{d-1}$. This corresponds to the case of "small oscillations" considered by BKL [BKL70, BKL82], for which they verify (in 3 dimensions) that the evolution is indeed controlled by the $\alpha_{i j k}$-terms even in that region. (ii) For the Kasner solution, one has $\exp \left(-2 \mu_{d}\right) \simeq t^{1-p^{d}} \rightarrow 0$ and so the Kasner solution never reaches the region where $\mu_{d}$ is arbitrarily negatively large.
} 
2. The potential $V_{\phi}=g g^{i j} \partial_{i} \phi \partial_{j} \phi$ of the dilaton has the same form as the subdominant gravitational terms that we have just neglected, since the exponentials that control its asymptotic behaviour are also $\exp \left[-2 \mu_{j}(\beta)\right]$. Consequently, we can drop $V_{\phi}$ in the BKL limit.

3. The above curvature computation involves only the Cartan formulas. It would also hold true if $\theta^{k}=\sum_{i} \mathcal{N}_{k i} f^{i}$, where $f^{i}=f_{j}^{i}(x) d x^{j}$ is an arbitrary fixed frame. The structure functions $C_{m n}^{i}(x)$ would get contributions from both $\mathcal{N}_{k i}$ (and its spatial derivatives) and $f^{i}$. We have taken above $f_{j}^{i}=\delta_{j}^{i}$. Then, $C^{i}{ }_{m n}=C^{i}{ }_{m n}(\mathcal{N})$. The other extreme case is $\mathcal{N}_{k i}=\delta_{i j}$, in which case $C_{m n}^{i}=C_{m n}^{i}(f)$. In fact, not all gravitational walls $\alpha_{i j k}$ appear if we make the choice $f^{i}=d x^{i}$ since it then follows from the formulas (3.9) that $C_{i j}^{d}=0\left(i, j\right.$ arbitrary), $C_{i j}^{d-1}=0$ (with both $i, j \neq d)$ etc. Hence, $\left(C_{i j}^{d}\right)^{2}=0$ and the gravitational walls $\alpha_{d i j}$ are absent. Similarly, only the gravitational walls $\alpha_{d-1 i d}$ are present among the walls $\alpha_{d-1 i j}$. To get all the gravitational walls, one needs a non-holonomic frame $f^{i}$. The dominant gravitational wall $\alpha_{1 d-1 d}$ is, however, always present, and this is what matters for the billiard (when gravitational walls are relevant at all).

4. The coefficients of the dominant exponentials involve only the undifferentiated structure functions $C^{i}{ }_{j k}$. Thus, one can mimic at each point $x$ the gravitational potential in the context of spatially homogeneous cosmologies, where the $C^{i}{ }_{j k}$ are constant, by considering homogeneity groups that are "sufficiently" non-Abelian so that none of the coefficients of the relevant exponentials vanishes (Bianchi types VIII and IX for $d=3$, other homogeneity groups for $d>3$ - see [DDRH88]).

\section{$4.4 p$-form walls}

\subsubsection{Electric walls}

We now turn to the electric potential $V_{(p)}^{e l}$, which we express in the basis $\left\{\theta^{k}\right\}$. One has

$$
V_{(p)}^{e l}=\frac{p !}{2} \sum_{i_{1}, i_{2}, \cdots, i_{p}} e^{-2 e_{i_{1} \cdots i_{p}}(\beta)}\left(\mathcal{E}_{(p)}^{i_{1} \cdots i_{p}}\right)^{2}
$$


where $\mathcal{E}^{i_{1} \cdots i_{p}}$ are the components of the electric field $\pi_{(p)}^{m_{1} \cdots m_{p}}$ in the basis $\left\{\theta^{k}\right\}$,

$$
\mathcal{E}_{(p)}^{i_{1} \cdots i_{p}}=\sum_{m_{1}, \cdots, m_{p}} \mathcal{N}_{i_{1} m_{1}} \mathcal{N}_{i_{2} m_{2}} \cdots \mathcal{N}_{i_{p} m_{p}} \pi_{(p)}^{m_{1} \cdots m_{p}}
$$

and where $e_{i_{1} \ldots i_{p}}(\beta)$ are the electric linear forms

$$
e_{i_{1} \cdots i_{p}}(\beta)=\beta^{i_{1}}+\cdots+\beta^{i_{p}}+\frac{\lambda^{(p)}}{2} \phi \quad\left(\text { all } i_{j} \text { 's distinct }\right)
$$

(the indices $i_{j}$ 's are all distinct because $\mathcal{E}_{(p)}^{i_{1} \cdots i_{p}}$ is completely antisymmetric). The variables $\mathcal{E}^{i_{1} \cdots i_{p}}$ do not depend on the $\beta^{\mu}$. It is thus rather easy to take the BKL limit. The exponentials in (4.39) are multiplied by positive factors which are different from zero in the generic case. Thus, in the BKL limit, $V_{(p)}^{e l}$ becomes

$$
V_{(p)}^{e l} \simeq \sum_{i_{1}<i_{2}<\cdots<i_{p}} \Theta\left[-2 e_{i_{1} \cdots i_{p}}(\beta)\right]
$$

Note that the transformation from the variables $\left(\mathcal{N}_{i j}, P_{i j}, A_{m_{1} \cdots m_{p}}^{(p)}, \pi_{(p)}^{m_{1} \cdots m_{p}}\right)$ to the variables $\left(\mathcal{N}_{i j}, P_{i j}, \mathcal{A}_{m_{1} \cdots m_{p}}^{(p)}, \mathcal{E}_{(p)}^{i_{1} \cdots i_{p}}\right)$ is a point canonical transformation whose explicit form is obtained from

$$
\sum_{i<j} P_{i j} \dot{\mathcal{N}}_{i j}+\sum_{p} \pi_{(p)}^{m_{1} \cdots m_{p}} \dot{A}_{m_{1} \cdots m_{p}}^{(p)}=\sum_{i<j} P_{i j}^{\prime} \dot{\mathcal{N}}_{i j}+\sum_{p} \mathcal{E}_{(p)}^{i_{1} \cdots i_{p}} \dot{\mathcal{A}}_{m_{1} \cdots m_{p}}^{(p)}
$$

(The momenta $P_{i j}$ conjugate to $\mathcal{N}_{i j}$ get redefined by terms involving $\mathcal{E}, \mathcal{N}$ and $\mathcal{A}$ since the components $\mathcal{A}_{m_{1} \cdots m_{p}}^{(p)}$ of the $p$-forms in the basis $\left\{\theta^{k}\right\}$ involve the $\mathcal{N}$ 's,

$$
\mathcal{A}_{i_{1} \cdots i_{p}}^{(p)}=\sum_{m_{1}, \cdots, m_{p}}\left(\mathcal{N}^{-1}\right)_{m_{1} i_{1}} \cdots\left(\mathcal{N}^{-1}\right)_{m_{p} i_{p}} A_{(p) m_{1} \cdots m_{p}}
$$

This does not affect the symmetry walls in the BKL limit.)

\subsubsection{Magnetic walls}

The magnetic potential is dealt with similarly. Expressing it in the $\left\{\theta^{k}\right\}$ frame, one obtains

$$
V_{(p)}^{\text {magn }}=\frac{1}{2(p+1) !} \sum_{i_{1}, i_{2}, \cdots, i_{p+1}} e^{-2 m_{i_{1} \cdots i_{p+1}}(\beta)}\left(\mathcal{F}_{(p) i_{1} \cdots i_{p+1}}\right)^{2}
$$


where $\mathcal{F}_{(p) i_{1} \cdots i_{p+1}}$ are the components of the magnetic field $F_{(p) m_{1} \cdots m_{p+1}}$ in the basis $\left\{\theta^{k}\right\}$,

$$
\mathcal{F}_{(p) i_{1} \cdots i_{p+1}}=\sum_{m_{1}, \cdots, m_{p+1}}\left(\mathcal{N}^{-1}\right)_{m_{1} i_{1}} \cdots\left(\mathcal{N}^{-1}\right)_{m_{p+1} i_{p+1}} F_{(p) m_{1} \cdots m_{p+1}}
$$

and where $m_{i_{1} \ldots i_{p+1}}(\beta)$ are the magnetic linear forms

$$
m_{i_{1} \cdots i_{p+1}}(\beta)=\sum_{j \notin\left\{i_{1}, i_{2}, \cdots i_{p+1}\right\}} \beta^{j}-\frac{\lambda^{(p)}}{2} \phi
$$

(all $i_{j}$ 's are distinct). One sometimes rewrites $m_{i_{1} \cdots i_{p+1}}(\beta)$ as $b_{i_{p+2} \cdots i_{d}}$, where $\left\{i_{p+2}, i_{p+3}, \cdots, i_{d}\right\}$ is the set complementary to $\left\{i_{1}, i_{2}, \cdots i_{p+1}\right\}$; e.g.,

$$
b_{12 \cdots d-p-1}=\beta^{1}+\cdots+\beta^{d-p-1}-\frac{\lambda^{(p)}}{2} \phi=m_{d-p \cdots d}
$$

The exterior derivative $\mathcal{F}$ of $\mathcal{A}$ in the non-holonomic frame $\left\{\theta^{k}\right\}$ involves of course the structure coefficients, i.e., of frame $\mathcal{F}_{(p) i_{1} \cdots i_{p+1}}=\partial_{\left[i_{1}\right.} \mathcal{A}_{\left.i_{2} \cdots i_{p+1}\right]}+$ " $C \mathcal{A}$ "-terms where $\partial_{i_{1}} \equiv \sum_{m_{1}}\left(\mathcal{N}^{-1}\right)_{m_{1} i_{1}}\left(\partial / \partial x^{m_{1}}\right)$ is here the frame derivative.

Again, the BKL limit is quite simple and yields (assuming generic magnetic fields)

$$
V_{(p)}^{\text {magn }} \simeq \sum_{i_{1}<\cdots<i_{d-p-1}} \Theta\left[-2 b_{i_{1} \cdots i_{d-p-1}}(\beta)\right]
$$

Note that, just as the off-diagonal variables, the electric and magnetic fields get frozen in the BKL limit since the Hamiltonian no longer depends on the $p$-form variables. These drop out because one can rescale the coefficient of any $\Theta$-function to be one (when it is not zero), absorbing thereby the dependence on the $p$-form variables.

The scale factors are constrained by the further conditions

$$
e_{i_{1} \cdots i_{p}}(\beta) \geq 0, \quad b_{i_{1} \cdots i_{d-p-1}}(\beta) \geq 0 .
$$

The boundary hyperplanes $e_{i_{1} \cdots i_{p}}(\beta)=0$ and $b_{i_{1} \cdots i_{d-p-1}}(\beta)=0$ are called the "electric" and "magnetic" walls, respectively. 


\subsection{Billiards}

At this point, we see that the interesting dynamics as one goes towards the spacelike singularity is carried by the scale factors since the off-diagonal variables (including the $p$-form variables) asymptotically drop out. Furthermore, the evolution of the scale factors at each spatial point is, in the BKL limit, a broken null straight line of the metric $G_{\mu \nu} d \beta^{\mu} d \beta^{\nu}$ interrupted by collisions again the sharp walls

$$
w_{A}(\beta) \equiv w_{A \mu} \beta^{\mu}=0
$$

defined by the symmetry, gravitational and $p$-form potentials through $V=$ $\sum_{A} \Theta\left(-2 w_{A}(\beta)\right)$. All these walls are timelike hyperplanes. Indeed, we have found above that the gradients to the symmetry walls have squared norm equal to 2, see Eq. (3.18). Similarly, the gradients to the gravitational walls $\alpha_{i j k}=0$ are spacelike and, in fact, have the same squared norm +2 . Finally, the gradients to the electric wall $e_{i_{1} \cdots i_{p}}$ have also positive squared norm

$$
\frac{p(d-p-1)}{d-1}+\left(\frac{\lambda^{(p)}}{2}\right)^{2}
$$

which is equal to the squared norm of the corresponding magnetic walls. Incidentally, this shows that the norm of the $p$-form walls is invariant under electric-magnetic duality.

Because the walls are timelike, the velocity undergoes under a collision a geometric reflection in the hyperplane, which is an element of the orthochronous Lorentz group. The reflection preserves the norm and the timeorientation (hence, the velocity vector remains null and future-oriented).

The billiard is obtained by radial projection onto hyperbolic space. The billiard ball is constrained to be in the region $w_{A}(\beta) \geq 0$. Not all the walls are relevant since some of the inequalities $w_{A}(\beta) \geq 0$ are consequences of the others [DH01]. Only the dominant wall forms, in terms of which all the other wall forms can be expressed as linear combinations with non-negative coefficients, are relevant for determining the billiard. Usually, these are the symmetry walls and some of the $p$-form walls. The billiard is in general non-compact because some walls meet at infinity. However, even when it is non-compact, it can have finite volume.

In fact, the geodesic motion in a billiard in hyperbolic space has been much studied. It is known that this motion is chaotic or non-chaotic according to whether the billiard has finite or infinite volume [Ma69, HM79, Z84, EMcM93]. 


\section{Notes:}

1. Because of reparametrization invariance - in particular, time redefinitions -, some indicators of chaos must be used with care in general relativity, see [CL96, IM01] for a discussion of the original mixmaster model.

2. The hyperbolic billiard description of the $(3+1)$-dimensional mixmaster system was first worked out by Chitre [C72] and Misner [M94]. Our derivation of the asymptotic expression for the potential follows [K93, IKM94]. The extension to higher dimensions with perfect fluid sources was considered in [KM94], without symmetry walls. Exterior form sources were investigated in [IM95, IM99] for special classes of metric and $p$-form configurations.

\subsection{Constraints}

We have just seen that in the BKL limit, the evolution equations become ordinary differential equations with respect to time. Although the spatial points are decoupled in the evolution equations, they are, however, still coupled in the constraints. These constraints just restrict the initial data and need only be imposed at one time, since they are preserved by the dynamical equations of motion. Indeed, one easily finds that

$$
\dot{\mathcal{H}}=0
$$

since $\left[\mathcal{H}(x), \mathcal{H}\left(x^{\prime}\right)\right]=0$ in the ultralocal limit. This corresponds simply to the fact that the collisions preserve the lightlike character of the velocity vector. Furthermore, the gauge constraints (4.9) are also preserved in time since the Hamiltonian constraint is gauge-invariant. Finally, the momentum constraint fulfills

$$
\dot{\mathcal{H}}_{k}(x)=\partial_{k} \mathcal{H} \approx 0
$$

It is important to observe that the restrictions on the initial data do not constrain the coefficients of the walls in the sense that these may assume non-zero values. For instance, it is well known that it is consistent with Gauss law to take non-vanishing electric and magnetic energy densities; thus the coefficients of the electric and magnetic walls are indeed generically nonvanishing. In fact, the constraints are conditions on the spatial gradients of the variables entering the wall coefficients, not on these variables themselves. 
In some non-generic contexts, however, the constraints could force some of the wall coefficients to be zero; the corresponding walls would thus be absent. [E.g., for vacuum gravity in four dimensions, the momentum constraints for some Bianchi homogeneous models force some symmetry wall coefficients to vanish. But this is peculiar to the homogeneous case.]

It is easy to see that the number of arbitrary physical functions involved in the solution of the BKL equations of motion is the same as in the general solution of the complete Einstein-matter equations. Indeed, the number of constraints on the initial data and the residual gauge freedom are the same in both cases. Further discussion of the constraints in the BKL context may be found in [AR01, DHRW02].

\subsection{Chern-Simons or Yang-Mills couplings, Chapline- Manton terms}

The addition of Chern-Simons terms, Yang-Mills or Chapline-Manton couplings does not modify the billiard picture and, furthermore, does not bring in new walls. The only change in the asymptotic dynamics is a modification of the constraints.

\section{Yang-Mills couplings}

We start with the Yang-Mills coupling terms. The contribution to the energy density from the Yang-Mills field takes the same form as for a collection of abelian 1-forms, with the replacement of the momenta $\mathcal{E}^{i}$ by the Yang-Mills momenta $\mathcal{E}_{a}^{i}, a=1, \cdots, N$ (with $N$ the dimension of the internal Lie algebra) and of the magnetic fields by the corresponding non-abelian field strengths. As their abelian counterparts, these do not involve the scale factors $\beta^{\mu}$. Because of this key property, the same analysis goes through. Each electric and magnetic 1-form wall is simply repeated a number of times equal to the dimension of the Lie algebra. Gauss law is, however, modified and reads now:

$$
\mathcal{D}_{i} \mathcal{E}_{a}^{i} \equiv \nabla_{i} \mathcal{E}_{a}^{i}+f^{b}{ }_{a c} \mathcal{E}_{b}^{i} A_{i}^{c}=0 .
$$

Here, $\nabla_{i}$ is the standard metric covariant derivative. Similarly, the momentum constraints are modified and involves the non-abelian field strengths.

\section{Chapline-Manton couplings and Chern-Simons terms}

The discussion of Chapline-Manton couplings or Chern-Simons terms proceeds in the same way. The energy-density of the $p$-forms has the same de- 
pendence on the scale factors as in the absence of couplings, i.e., provides the same exponentials. The only difference is that the wall coefficients are different functions of the $p$-form canonical variables; but this difference is again washed out in the sharp wall limit, where the coefficients can be replaced by one (provided they are non zero). The momentum and Gauss constraints are genuinely different and impose different conditions on the initial data.

Note: although Chern-Simons terms do not generically change the features of the billiard (shape, volume), they may play a more significant rôle in peculiar contexts where only specialized field configurations are considered. This occurs for instance in [IM99], where it is shown that the 11-dimensional supergravity Chern-Simons term, in the case of spatially homogeneous metrics and magnetic fields, may constrain some electromagnetic walls to "accidentally" disappear. This makes the otherwise finite-volume billiard to be of infinite volume.

\section{$5 \quad$ Velocity-dominance - Strong coupling/small tension limit}

It is sometimes useful to separate in the Hamiltonian the time derivatives (conjugate momenta) from the space derivatives. This yields

$$
\mathcal{H}=K^{\prime}+\varepsilon V^{\prime}
$$

where $\varepsilon= \pm 1$ according to whether the spacetime signature is Lorentzian $(\varepsilon=1)$ or Euclidean $(\varepsilon=-1)$. Here,

$$
K^{\prime}=K+V_{S}+V_{(p)}^{e l}
$$

and

$$
V^{\prime}=V_{G}+V_{(p)}^{\text {magn }}+V_{\phi} .
$$

The reason that this splitting is useful is that for some models, the asymptotic dynamics is entirely controlled by $K^{\prime}$, i.e., by the limit $\varepsilon=0$. This occurs whenever the billiard that emerges in the BKL limit is defined by the symmetry and electric walls, as it happens for instance for eleven-dimensional supergravity [DH00a], or the pure Einstein-Maxwell system in spacetime dimensions $D \geq 5$ [DH00b, KS00]. Curvature and magnetic walls are then subdominant, i.e., spatial gradients become negligible as one goes toward the singularity. 
If the curvature and magnetic walls can indeed be neglected, the evolution equations are exactly the same as the equations of motion obtained by performing a direct torus reduction to $1+0$ dimensions. We stress, however, that no homogeneity assumption has ever been made. This effective torus dimensional reduction follows from the dynamics and is not imposed by hand.

The limit $\varepsilon=0$ is known as the "zero signature limit" [T78] and lies half-way between spacetimes of Minkowskian or Lorentzian signature. It corresponds to a vanishing velocity of light (or vanishing "medium tension"); the underlying geometry is built on the Carroll contraction of the Lorentz group [H79]. [The terminology "strong coupling" is also used [I76] and stems from the fact that with appropriate redefinitions, $\mathcal{H}$ can formally be rewritten as $\mathcal{H}=G K^{\prime}+(1 / G) V^{\prime}$ where $G$ is Newton's constant.] A revival of interest in this ultrarelativistic limit has arisen recently [D98, LS01, A02].

When the billiard has infinite volume, the dynamics in the vicinity of the singularity is even simpler because there is only a finite number of collisions with the walls. The system generically settles after a finite time in a Kasner-like motion that lasts all the way to the singularity. The asymptotic dynamics is controlled solely by the kinetic energy $K$ of the scale factors (after all collisions have taken place). This case, where both spatial gradients and matter (here $p$-form) terms can be neglected, has been called "velocitydominated" in [E72] and enables a rigorous analysis of its asymptotic dynamics by means of "Fuchsian" techniques [AR01, DHRW02]. By contrast, rigorous results concerning the finite-volume case are rare (see, however, the recent analytic advances in [R01]). Besides the existing rigorous results, one should also mention the good wealth of numerical support of the BKL ideas [BGIMW98, B02].

\section{Miscellany and Conclusions}

In this paper, we have shown that theories involving gravity admits a remarkable asymptotic description in the vicinity of a spacelike singularity in terms of billiards in hyperbolic space. Depending on whether the actual billiard has finite or infinite volume, the dynamical evolution of the local scale factors is chaotic and of the mixmaster type, or monotone and Kasner-like. The billiard, and in particular its volume, is a fundamental characteristic of the theory, in the sense that it is determined solely by the field content and the parameters in the Lagrangian, and not by the initial 
conditions (in the generic case; i.e., there may be initial conditions for which some walls are absent - and the billiard is changed -, but these are exceptional). Although we have not investigated the physical implications of this property for cosmological scenarios (in particular, string-inspired cosmologies [GV92, BDV98, LWC00, W02, GV02]), nor its quantum analog, we believe that this result is already interesting in its own right because it uncovers an intrinsic feature of gravitational theories. Furthermore, the regularity properties of the billiards appear to give a powerful access to hidden symmetries, as we now briefly discuss.

\subsection{Kac-Moody billiards}

The billiard description holds for all systems governed by the action (1.1). In general, the billiard has no notable regularity property. In particular, the dihedral angles between the faces, which depend on the (continuous) dilaton couplings (when there is a dilaton), need not be integer submultiples of $\pi$. In some instances, however, the billiard can be identified with the fundamental Weyl chamber of a symmetrizable Kac-Moody algebra of indefinite type, with Lorentzian signature metric [DH01]. One then says that the billiard is a "Kac-Moody billiard". [See [Kac90, MP95] for information on KacMoody (KM) algebras.] In [DH01], superstring models were considered and the rank $10 \mathrm{KM}$ algebras $E_{10}$ and $B E_{10}$ were shown to emerge, in line with earlier conjectures made in $[\mathrm{J} 80, \mathrm{~J} 85]^{9}$. This was then further extended to pure gravity in any number of spacetime dimensions, for which the relevant $\mathrm{KM}$ algebra is $A E_{d}$, and it was understood that chaos (finite volume of the billiard) is equivalent to hyperbolicity of the underlying KM algebra [DHJN01]. The original case of $D=4$ pure gravity corresponds to the hyperbolic algebra $A E_{3}$ investigated in $[\mathrm{FF} 83, \mathrm{~N} 92]^{10}$. Further examples of emergence of Lorentzian KM algebras, based on the models of [BMG88, CJLP99], are given in [DdBHS02].

The walls that determine the billiards are the dominant walls. For KM billiards, they correspond to the simple roots of the KM algebra. The sub-

\footnotetext{
${ }^{9}$ Note that the Weyl groups of the $E$-family have been discussed in a similar vein in the context of $U$-duality [LPS96, OPR98].

${ }^{10}$ Note that in the original analysis of [BKL70, BKL82, C72, M94], the symmetry walls are not included; the KM algebra that arises has a $3 \times 3$ Cartan matrix given by $A_{i i}=2$, $A_{i j}=-2(i \neq j)$ and its fundamental Weyl chamber (radially projected on $H_{2}$ ) is the ideal equilateral triangle having its 3 vertices at infinity.
} 
dominant walls also have an algebraic interpretation in terms of higher height positive roots [DHN02]. This enables one to go beyond the BKL limit and to see the beginning of a possible identification of the dynamics of the scale factors and of all the off-diagonal variables (including the $p$-form variables) with that of a non-linear sigma model obtained by formally taking the coset of the KM group with its maximal compact subgroup [DHN02].

\subsection{Chaos versus non-chaos}

We close our paper by indicating when the models described by the action (1.1) exhibit mixmaster behaviour (finite volume billiard) or Kasner-like behaviour (infinite volume billiard) (for generic initial conditions).

- Pure gravity billiards have finite volume for spacetime dimension $D \leq$ 10 and infinite volume for spacetime dimension $D \geq 11$ [DHS85]. This can be understood in terms of the underlying Kac-Moody algebra [DHJN01].

- Gravity + dilaton has always an infinite volume billiard [BK73].

- Gravity $+p$-forms (with $p \neq 0$ and $p<D-2$ ) and no dilaton has a finite volume billiard [DH00b]. In particular, 11-dimensional supergravity exhibits mixmaster behaviour, while vacuum gravity in 11 dimensions does not. The 3 -form is crucial for closing the billiard. Similarly, the Einstein-Maxwell system in 4 (in fact any number of) dimensions has an finite-volume billiard (see [J86, L97, W99] for a discussion of four-dimensional homogeneous models with Maxwell field exhibiting mixmaster behaviour).

- The volume of the mixed Einstein-dilaton- $p$-form system depends on the dilaton couplings. For a given spacetime dimension $D$ and a given menu of $p$-forms there exists a "subcritical" domain $\mathcal{D}$ in the space of of the dilaton couplings (an open neighbourhood of the origin $\lambda^{(p)}=0$ ) such that: (i) when the dilaton couplings $\lambda^{(p)}$ belong to $\mathcal{D}$ the general behaviour is Kasner-like, but (ii) when the $\lambda^{(p)}$ do not belong to $\mathcal{D}$ the behaviour is oscillatory [DH00a, DHRW02]. For all the superstring models, the dilaton couplings do not belong to the critical domain and the billiard has finite volume. 


\section{Acknowledgements}

M.H. is grateful to the organizers of the "School on Quantum Gravity" held at CECS Valdivia, Chile (January 2002), where some of the material contained in this article was presented. He is also grateful to Claudio Teitelboim for numerous discussions on the zero-velocity-of-light limit, which arose his interest in the BKL analysis. The work of M.H. is supported in part by the "Actions de Recherche Concertées" of the "Direction de la Recherche Scientifique - Communauté Française de Belgique", by a "Pôle d'Attraction Interuniversitaire" (Belgium), by IISN-Belgium (convention 4.4505.86), by Proyectos FONDECYT 1970151 and 7960001 (Chile) and by the European Commission RTN programme HPRN-CT-00131, in which he is associated to K. U. Leuven. H.N. is partially supported by the EU contract HPRN-CT2000-00122. Both M.H. and H.N. would like to thank I.H.E.S. for hospitality while this work was carried out.

\section{A Iwasawa decomposition and asymptotics of non-diagonal $3 d$ Kasner metric}

The Iwasawa decomposition for three-dimensional metrics has been explicitly analyzed in [HPT82]. Setting

$$
\mathcal{N}=\left(\begin{array}{ccc}
1 & n_{1} & n_{2} \\
0 & 1 & n_{3} \\
0 & 0 & 1
\end{array}\right)
$$

together with

$$
\mathcal{A}=\left(\begin{array}{ccc}
\exp \left(-\beta^{1}\right) & 0 & 0 \\
0 & \exp \left(-\beta^{2}\right) & 0 \\
0 & 0 & \exp \left(-\beta^{3}\right)
\end{array}\right)
$$

one finds

$$
\begin{aligned}
& g_{11}=e^{-2 \beta^{1}}, \quad g_{12}=n_{1} e^{-2 \beta^{1}}, \quad g_{13}=n_{2} e^{-2 \beta^{1}} \\
& g_{22}=\left(n_{1}\right)^{2} e^{-2 \beta^{1}}+e^{-2 \beta^{2}}, \quad g_{23}=n_{1} n_{2} e^{-2 \beta^{1}}+n_{3} e^{-2 \beta^{2}} \\
& g_{33}=\left(n_{2}\right)^{2} e^{-2 \beta^{1}}+\left(n_{3}\right)^{2} e^{-2 \beta^{2}}+e^{-2 \beta^{3}}
\end{aligned}
$$


from which one gets

$$
\begin{aligned}
& \beta^{1}=-\frac{1}{2} \ln g_{11}, \quad \beta^{2}=-\frac{1}{2} \ln \left[\frac{g_{11} g_{22}-g_{12}^{2}}{g_{11}}\right], \\
& \beta^{3}=-\frac{1}{2} \ln \left[\frac{g}{g_{11} g_{22}-g_{12}^{2}}\right], \quad n_{1}=\frac{g_{12}}{g_{11}} \\
& n_{2}=\frac{g_{13}}{g_{11}}, \quad n_{3}=\frac{g_{23} g_{11}-g_{12} g_{13}}{g_{11} g_{22}-g_{12}^{2}} .
\end{aligned}
$$

On the other hand, (3.1) yields

$$
g_{i j}=t^{2 p^{1}} l_{i} l_{j}+t^{2 p^{2}} m_{i} m_{j}+t^{2 p^{3}} r_{i} r_{j}
$$

with

$$
L=\left(\begin{array}{ccc}
l_{1} & l_{2} & l_{3} \\
m_{1} & m_{2} & m_{3} \\
r_{1} & r_{2} & r_{3}
\end{array}\right) .
$$

Combining these relations, one obtains the time dependence of the Iwasawa variables

$$
\begin{aligned}
& \beta^{1}=-\frac{1}{2} \ln X, \beta^{2}=-\frac{1}{2} \ln \left[\frac{Y}{X}\right], \\
& \beta^{3}=-\frac{1}{2} \ln \left[\frac{t^{2\left(p^{1}+p^{2}+p^{3}\right)}(\operatorname{det} L)^{2}}{Y}\right], \\
& n_{1}=\frac{t^{2 p^{1}} l_{1} l_{2}+t^{2 p^{2}} m_{1} m_{2}+t^{2 p^{3}} r_{1} r_{2}}{X}, \\
& n_{2}=\frac{t^{2 p^{1}} l_{1} l_{3}+t^{2 p^{2}} m_{1} m_{3}+t^{2 p^{3}} r_{1} r_{3}}{X}, n_{3}=\frac{Z}{Y}
\end{aligned}
$$

with

$$
\begin{aligned}
X= & t^{2 p^{1}}\left(l_{1}\right)^{2}+t^{2 p^{2}}\left(m_{1}\right)^{2}+t^{2 p^{3}}\left(r_{1}\right)^{2}, \\
Y= & t^{2 p^{1}+2 p^{2}}\left(l_{1} m_{2}-l_{2} m_{1}\right)^{2}+t^{2 p^{1}+2 p^{3}}\left(l_{1} r_{2}-l_{2} r_{1}\right)^{2} \\
& \quad+t^{2 p^{2}+2 p^{3}}\left(m_{1} r_{2}-m_{2} r_{1}\right)^{2}, \\
Z= & t^{2 p^{1}+2 p^{2}}\left(l_{1} m_{2}-l_{2} m_{1}\right)\left(l_{1} m_{3}-l_{3} m_{1}\right) \\
& +t^{2 p^{1}+2 p^{3}}\left(l_{1} r_{2}-l_{2} r_{1}\right)\left(l_{1} r_{3}-l_{3} r_{1}\right) \\
& +t^{2 p^{2}+2 p^{3}}\left(m_{1} r_{2}-m_{2} r_{1}\right)\left(m_{1} r_{3}-m_{3} r_{1}\right) .
\end{aligned}
$$


Without loss of generality, one can assume $p^{1} \leq p^{2} \leq p^{3}$. If necessary, this can be achieved by multiplying $L$ by an appropriate permutation matrix. We shall in fact consider the case $p^{1}<p^{2}<p^{3}$, leaving the discussion of the limiting situations to the reader. One then finds, for generic $L$ 's $\left(l_{1} \neq 0\right.$, $\left.r_{1} \neq 0, l_{1} m_{2}-l_{2} m_{1} \neq 0, m_{1} r_{2}-m_{2} r_{1} \neq 0\right)$, the following asymptotic behaviour:

$$
\begin{array}{ll}
\tau \rightarrow-\infty: & \\
& \beta^{1} \sim p^{3} \tau, \beta^{2} \sim p^{2} \tau, \beta^{3} \sim p^{1} \tau, \\
& n_{1} \rightarrow \frac{r_{2}}{r_{1}}, n_{2} \rightarrow \frac{r_{3}}{r_{1}}, n_{3} \rightarrow \frac{m_{1} r_{3}-m_{3} r_{1}}{m_{1} r_{2}-m_{2} r_{1}} \\
\tau \rightarrow \infty: & \\
& \beta^{1} \sim p^{1} \tau, \beta^{2} \sim p^{2} \tau, \beta^{3} \sim p^{3} \tau, \\
& n_{1} \rightarrow \frac{l_{2}}{l_{1}}, n_{2} \rightarrow \frac{l_{3}}{l_{1}}, n_{3} \rightarrow \frac{l_{1} m_{3}-l_{3} m_{1}}{l_{1} m_{2}-l_{2} m_{1}} .
\end{array}
$$

It is rather clear that the $n_{i}$ 's should asymptotically tend to constants since they are homogeneous functions of degree zero in the $g_{i j}$ 's - in fact, ratios of polynomials of degree one or two in the $t^{2 p^{i}}$. It is a bit more subtle that the scale factors $\exp \left(-2 \beta^{i}\right)$, which are homogeneous of degree one in the $g_{i j}$ 's, are not all driven by the fastest growing term $\left(t^{2 p^{3}}\right.$ for $t \rightarrow \infty$ or $t^{2 p^{1}}$ for $\left.t \rightarrow 0^{+}\right)$. This is actually true only for the first scale factor $\exp \left(-2 \beta^{1}\right)$. The second scale factor $\exp \left(-2 \beta^{2}\right)$ feels the next subleading term $t^{2 p^{2}}$ because the fastest growing term drops from its numerator, equal to the minor $g_{11} g_{22}-\left(g_{12}\right)^{2}$. Similar cancellations occur for the last scale factor $\exp \left(-2 \beta^{3}\right)$, which feels only the smallest term $t^{2 p^{1}}(t \rightarrow \infty)$ or $t^{2 p^{3}}\left(t \rightarrow 0^{+}\right)$.

We come thus to the conclusion that the asymptotic behaviour of the Iwasawa variables is indeed simple: the scale factors asymptotically behave as the scale factors of the diagonal Kasner solutions, while the parameters $n_{i}$ parametrizing the off-diagonal components approach constants. The "out"values of the Kasner exponents differ from their "in"-values by a permutation such that the inequalities $\beta^{1} \leq \beta^{2} \leq \beta^{3}$ hold both for $\tau \rightarrow+\infty$ and $\tau \rightarrow-\infty$.

The frame $\{l, m, r\}$ where the spatial metric and the extrinsic curvature are simultaneously diagonal is called the "Kasner frame". Given that the time slicing has been fixed, this geometric frame is unique - up to individual normalization of each basis vector - when the eigenvalues of the extrinsic curvature are distinct, if one prescribes in addition some definite ordering 
of the eigenvalues (i.e. $p_{1}<p_{2}<p_{3}$ as above). For asymptotic values of $\tau$, the Iwasawa frames $\left\{\theta^{1}, \theta^{2}, \theta^{3}\right\}$ (which are by contrast not unique since one can redefine the coordinates $x^{i}$ ) become approximately time-independent and hence, the extrinsic curvature become approximately diagonal. Yet, the Iwasawa frames do not tend to align with the Kasner frame. To understand this point, we assume for definiteness that $\tau \rightarrow+\infty$. In that case, one gets from (A.19) that

$$
\theta^{1} \rightarrow \lambda l, \theta^{2} \rightarrow \mu l+\nu m, \quad \theta^{3} \rightarrow \varphi l+\chi m+\psi r
$$

where $\lambda=l_{1}^{-1}, \mu=-m_{1} /\left(l_{1} m_{2}-l_{2} m_{1}\right), \nu=l_{1} /\left(l_{1} m_{2}-l_{2} m_{1}\right)$, and $\varphi, \chi, \psi$ are some constants. This implies that the misalignment of the Iwasawa frames with respect to the Kasner frame is a small effect, in the sense that it induces a small change in the metric - even though the coefficients $\lambda, \mu, \nu, \varphi, \chi$ and $\psi$ are of order unity. Indeed, the change of frame $\{l, m, r\} \rightarrow\{l, m+\alpha l, r+$ $\beta l+\gamma m\}$, with $\alpha, \beta, \gamma$ constants $^{11}$, induces changes $\Delta g_{a b}$ which fulfill the smallness condition $\Delta g_{a b}<<\sqrt{g_{a a}} \sqrt{g_{b b}}$ (where $g_{a b}$ is the metric in the frame $\{l, m, r\})$. For instance, $\Delta g_{11} \sim t^{2 p_{2}}<<t^{2 p_{1}}$. Thus, it is perfectly consistent to find that the Iwasawa frames become asymptotically constant as $\tau \rightarrow+\infty$ without aligning with the principal axes of the extrinsic curvature. [Note that the extrinsic curvature becomes asymptotically diagonal in the Iwasawa frame precisely in the sense that the off-diagonal components fulfill $\left|K_{i j}\right|<<$ $\sqrt{\left|K_{i i}\right|} \sqrt{\left|K_{j j}\right|}(i \neq j)$ for large $\tau$. The mixed components $K_{j}^{i}$ tend to a non-diagonal matrix, however.]

\section{B Freezing the off-diagonal variables: a toy model}

We have seen in the text that the off-diagonal variables and the $p$-form variables get frozen to constant values in the BKL limit. We provide here a more detailed understanding of this property by discussing a simpler model which shares the same features.

Consider a system with two canonically conjugate pairs $(q, p),(Q, P)$ and

\footnotetext{
${ }^{11}$ In order to preserve diagonality of the metric, there are of course unwritten additional small correction terms to the new vectors; for instance, the new vector $l$ contains a term proportional to $m$ with coefficient $t^{-2\left(p_{1}-p_{2}\right)}$.
} 
time-dependent Hamiltonian

$$
H=\frac{1}{2} p^{2}+\frac{1}{2} P^{2} \rho^{k} e^{-\rho q} \quad(k \text { positive integer })
$$

where $\rho$ is $\rho \equiv \exp (T)$, with $T$ the time. One can think of $(q, p)$ as mimicking the scale factors, while $(Q, P)$ mimicks the off-diagonal components or the $p$-form variables. In (B.1), there is only one potential wall for $q$ (namely, the second term). We shall consider later the case with several walls.

One has $\dot{P}=0$, hence $P=P_{0}$ where $P_{0}$ is a constant which we assume to be different from zero. In the limit of large times, the motion in $q$ is a free motion interrupted by a collision against the potential wall,

$$
q=\left|T-T_{0}\right|+q_{0}
$$

where $T_{0}$ is the time of the collision and $q_{0}$ the turning point. We take a unit initial velocity. The location of the turning point is determined by

$$
P_{0}^{2} \rho_{0}^{k} e^{-\rho_{0} q_{0}}=1 \text {. }
$$

The time length $\Delta T$ of the collision is roughly of the order $1 / \rho_{0}$ : the later the collision, the sharper the wall. Let us evaluate the change in $Q$ in the collision. To that end, we need to compute

$$
\Delta Q=P_{0} \int_{-\infty}^{\infty} d T \rho^{k} e^{-\rho\left(\left|T-T_{0}\right|+q_{0}\right)}
$$

since $\dot{Q}=P \rho^{k} \exp (-\rho q)$. The integrand is maximum at $T=T_{0}$. We can approximate the integral by the value at the maximum times the time length of the collision. Using (B.3), one gets

$$
\Delta Q \approx \frac{1}{P_{0} \rho_{0}}
$$

Hence, the variable $Q$ receives a kick during the collision (which can be of order one at early times), but the later the collision, the smaller the kick.

Assume now that there is another wall with the same time dependence, say at $q=d$, so that $q$ bounces between these two walls,

$$
V_{\text {additional }}=\frac{1}{2} P^{2} \rho^{k} e^{-\rho(d-q)} .
$$


Because the speed of $q$ remains constant (in the large $T$ limit), the collisions are equally spaced in $T$. At each collision, $Q$ receives a kick of order $1 / \rho_{0}$. The total change in $Q$ is obtained by summing all the individual changes, which yields

$$
(\Delta Q)_{\text {Total }} \sim \sum_{n} e^{n d}
$$

(the time interval between two collisions is $d$ since we assumed unit velocity). This sum converges. After a while, one can neglect the further change in $Q$, i.e., assume $\dot{Q}=0$. The Hamiltonian describing the large time limit is obtained by taking the sharp wall limit in the above $H$, and reads therefore

$$
H=\frac{1}{2} p^{2}+\Theta(-q)+\Theta(q-d)
$$

The pair $(Q, P)$ drops out because it is asymptotically frozen. Our analysis justifies taking the sharp wall limit directly in $H$ for this system, which is the procedure we followed in the text to get the gravitational billiards.

\section{Kasner frame versus Iwasawa frames}

In the original analysis of BKL [BKL70, BKL82], the description of the evolution of the fields is not carried out in the Iwasawa frames defined algebraically using the Iwasawa decomposition, but rather in the geometrically defined Kasner frame where the spatial metric and the extrinsic curvature are simultaneously diagonal and the Kasner exponents are ordered (during the Kasner "epoch" under study). Belinskii, Khalatnikov and Lifshitz found that the Kasner axes undergo, under collisions with the gravitational walls, changes of order unity with respect to time-independent spatial frames (frames having zero Lie bracket with $\partial / \partial t$ ), no matter how close one gets to the singularity [BKL72]; therefore, the Kasner axes generically never come to rest if there is an infinite number of collisions. The purpose of this appendix is to reconcile this result with the above conclusion that the off-diagonal components $\mathcal{N}_{i j}$ tend to constants as one approaches the singularity. The key point is, of course, that Kasner frames and Iwasawa frames do not coincide in general. We shall treat explicitly the $3+1$-case.

In the Kasner frame, the metric takes the form of Eq. (A.9) with $t^{2 p_{1}}, t^{2 p_{2}}$ and $t^{2 p_{3}}$ replaced respectively by $a^{2}, b^{2}$ and $c^{2}$. Far from the gravitational walls, the functions $a^{2}, b^{2}$ and $c^{2}$ are given by $t^{2 p_{1}}, t^{2 p_{2}}$ and $t^{2 p_{3}}$ where $p_{1}, p_{2}$ 
and $p_{3}$ depend only on the spatial coordinates, and the frame components $l_{i}, m_{i}$ and $n_{i}$ are also time-independent. Neither of these properties hold in the collision region. The collision against the gravitational wall induces the familiar transition between the Kasner exponents

$$
p_{1}^{\prime}=\frac{-p_{1}}{1+2 p_{1}}, p_{2}^{\prime}=\frac{p_{2}+2 p_{1}}{1+2 p_{1}}, p_{3}^{\prime}=\frac{p_{3}+2 p_{1}}{1+2 p_{1}}
$$

and also a change in the frame components given by [BKL72]

$$
l_{i}^{\prime}=l_{i}, \quad m_{i}^{\prime}=m_{i}+\sigma_{m} l_{i}, \quad r_{i}^{\prime}=r_{i}+\sigma_{r} l_{i}
$$

where $\sigma_{m}$ and $\sigma_{r}$ are of order unity. This formula holds for pure gravitational collisions (far from the symmetry walls), i.e., under the assumption that both $b^{2}$ and $c^{2}$ are very small compared with $a^{2}$ [BKL72] so that only one term in the potential is non-negligible, namely, the curvature term proportional to $a^{4}$ (the only case we shall consider explicitly). To avoid "interference" with the symmetry wall $b^{2}=c^{2}$, we impose also the condition $c^{2}<<b^{2}$, although this is actually not necessary for showing that the Iwasawa parameters $n_{i}$ are constant. Now, under the assumption $b^{2}<<a^{2}$ and $c^{2}<<b^{2}$, it is easy to see, using the formulas (A.11) through (A.17) with $t^{2 p_{1}}, t^{2 p_{2}}$ and $t^{2 p_{3}}$ replaced by $a^{2}, b^{2}$ and $c^{2}$, that $n_{1}, n_{2}$ and $n_{3}$ are respectively given by

$$
n_{1}=\frac{l_{2}}{l_{1}}, n_{2}=\frac{l_{3}}{l_{1}}, n_{3}=\frac{l_{1} m_{3}-l_{3} m_{1}}{l_{1} m_{2}-l_{2} m_{1}}
$$

(just like in (A.19)). It is clear that if we substitute in these formulas $l_{i}^{\prime}$, $m_{i}^{\prime}$ and $r_{i}^{\prime}$ for $l_{i}, m_{i}$ and $r_{i}$ according to (C.2), we get no change in the offdiagonal variables $n_{1}, n_{2}$ and $n_{3}$, as we wanted to show. There is thus no contradiction between the change of Kasner axes and the freezing of the offdiagonal Iwasawa variables. The same conclusion holds for collisions against the other types of walls, where the Kasner axes "rotate" as in (C.2).

\section{Hamiltonian reduction}

We provide in this appendix a derivation of the BKL limit through a (partial) Hamiltonian reduction of the dynamics, along the lines discussed in subsection 2.2 for homogeneous models of Bianchi type I. ["Partial" because we only take care of the Hamiltonian constraint; the other constraints must still be imposed.] Our considerations are of a purely local nature. 
We proceed as in section 4 but, to begin with, we impose only the orthogonal gauge condition $N^{i}=0$, without fixing the lapse. The metric reads thus

$$
d s^{2}=-(\tilde{N} \sqrt{g})^{2}\left(d x^{0}\right)^{2}+g_{i j}\left(x^{0}, x^{i}\right) d x^{i} d x^{j} .
$$

The action is, in terms of the Iwasawa variables,

$$
\begin{aligned}
& S\left[\beta^{\mu}, \mathcal{N}_{i j}, \pi_{\mu}, P_{i j}, A_{m_{1} \cdots m_{p}}^{(p)}, \pi_{(p)}^{m_{1} \cdots m_{p}}\right]= \\
& \int d x^{0}\left(\int d^{d} x\left(\pi_{\mu} \dot{\beta}^{\mu}+\sum_{i<j} P_{i j} \dot{\mathcal{N}}_{i j}+\sum_{p} \pi_{(p)}^{m_{1} \cdots m_{p}} \dot{A}_{m_{1} \cdots m_{p}}^{(p)}\right)-H\right)(\mathrm{D} .2)
\end{aligned}
$$

where the Hamiltonian is

$$
H=\int d^{d} x \tilde{N} \mathcal{H}
$$

(with $\mathcal{H}$ given by $(4.12)$ ).

As explained in the text, our main assumption is that $\beta^{\mu} \beta_{\mu}$ is $<0$ for large $x^{0}$ and that $\rho^{2}=-\beta^{\mu} \beta_{\mu}$ monotonously tends to $+\infty$ as $x^{0} \rightarrow+\infty$ (once $x^{0}$ is big enough). In terms of $T \equiv \ln \rho$ and the hyperboloid variables $\gamma^{\mu}$ (constrained by $\gamma^{\mu} \gamma_{\mu}=-1$ ), the action is

$$
\begin{aligned}
& S\left[T, \gamma^{\mu}, \mathcal{N}_{i j}, \pi_{T}, \tilde{\pi}_{\mu}, P_{i j}, A_{m_{1} \cdots m_{p}}^{(p)}, \pi_{(p)}^{m_{1} \cdots m_{p}}\right]= \\
& \int d x^{0}\left(\int d^{d} x\left(\pi_{T} \dot{T}+\tilde{\pi}_{\mu} \dot{\gamma}^{\mu}+\sum_{i<j} P_{i j} \dot{\mathcal{N}}_{i j}+\sum_{p} \pi_{(p)}^{m_{1} \cdots m_{p}} \dot{A}_{m_{1} \cdots m_{p}}^{(p)}\right)-H\right)
\end{aligned}
$$

with

$$
\begin{aligned}
H & =\int d^{d} x \frac{\tilde{N}}{4 \rho^{2}} \tilde{\mathcal{H}} \\
\tilde{\mathcal{H}} & =-\pi_{T}^{2}+\left(\tilde{\pi}_{\mu}\right)^{2}+4 \rho^{2}\left(V_{S}+V_{G}+V_{\phi}+\sum_{p} V_{(p)}\right)
\end{aligned}
$$

Here, $\tilde{\pi}_{\mu}$ are the (constrained) momenta conjugate to the hyperboloid variables. The coordinate $T$ is clearly a timelike variable in the space of the scale factors; large $T$ is the same as large $\sum_{i} \beta^{i}$ or small $g$, exactly as in the homogeneous case (the formulas are the same since the supermetric is ultralocal). 
Since $T$ is assumed to monotonously increase to $+\infty$ as $x^{0} \rightarrow+\infty$, we can use it as a time coordinate, i.e., impose the gauge condition $T=x^{0}$. The reduced action in that gauge is

$$
\begin{aligned}
& S\left[\gamma^{\mu}, \mathcal{N}_{i j}, \tilde{\pi}_{\mu}, P_{i j}, A_{m_{1} \cdots m_{p}}^{(p)}, \pi_{(p)}^{m_{1} \cdots m_{p}}\right]= \\
& \int d T\left[\int d ^ { d } x \left(\tilde{\pi}_{\mu} \frac{d \gamma^{\mu}}{d T}+\sum_{i<j} P_{i j} \frac{d \mathcal{N}_{i j}}{d T}\right.\right. \\
& \left.\left.+\sum_{p} \pi_{(p)}^{m_{1} \cdots m_{p}} \frac{d A_{m_{1} \cdots m_{p}}^{(p)}}{d T}\right)-H_{T}\right]
\end{aligned}
$$

where $H_{T}=-p_{T}$ is the Hamiltonian in the gauge $T=x^{0}($ for which $\dot{T}=1$ ),

$$
H_{T}=\sqrt{\left(\tilde{\pi}_{\mu}\right)^{2}+\rho^{2}\left(V_{S}+V_{G}+V_{\phi}+\sum_{p} V_{(p)}\right)} .
$$

$H_{T}$ is explicitly time-dependent (through $\rho=\exp (T)$ ).

So far, no approximation has been made. We now investigate the large time (large $T$ ) limit, which can be taken since $T$ is assumed to monotonously increase to $+\infty$. This is similar to investigating the large time dynamics of a Hamiltonian system with time-dependent Hamiltonian $H(q, p, t)$ by taking the $t \rightarrow \infty$ limit directly in the Hamiltonian (if it exists). As explained in the text, the potential becomes in that limit a sum of sharp wall potentials, so that one can replace (D.8) by

$$
H_{T}=\sqrt{\left(\tilde{\pi}_{\mu}\right)^{2}+\sum_{A} \Theta\left(-2 w_{A}(\gamma)\right)}
$$

which is time-independent. Because the walls are timelike and the free motion is lightlike, the asymptotic motion of the scale factors is a succession of futureoriented lightlike straight line segments and hence, it is indeed timelike. This provides a self-consistency check of the assumption that $\rho$ increases and tends to infinity.

Of course, the replacement of (D.8) by (D.9) is permissible only if the coefficients of the exponentials do not grow too fast, so that, as $T \rightarrow+\infty$, the variables $\mathcal{N}_{i j}, P_{i j}, A_{m_{1} \cdots m_{p}}^{(p)}$ and $\pi_{(p)}^{m_{1} \cdots m_{p}}$ as well as the spatial derivatives of the scale factors do not outgrow the exponentials. This other consistency check is also verified since we have shown that $\mathcal{N}_{i j}, P_{i j}, A_{m_{1} \cdots m_{p}}^{(p)}$ and $\pi_{(p)}^{m_{1} \cdots m_{p}}$ 
get asymptotically frozen. Similarly, from (2.17), one sees that, between collisions, $\partial \beta \sim \ln t$ and $\partial^{2} \beta \sim \ln t$ (with a coefficient of order one which changes in each collision) so that the terms $(\partial \beta)^{2}$ or $\partial^{2} \beta$ that multiply the subdominant gravitational walls do not outgrow the exponentials. [This is actually a bit trickier because it is not entirely clear that the coefficients of $\ln t$ remain of order one during collisions. This is because the evolution is independent at each spatial point, so that the $\beta$ 's might not remain differentiable and the spatial gradients might become more singular. This has been argued to lead to a kind of turbulent gravitational behaviour in which energy is pumped into shorter and shorter length scales [KK87, B92].]

Since the Hamiltonan does not depend explicitly on time, $\pi_{T} \equiv-H_{T}$ is constant in time. On the other hand, $\pi_{T}=\pi_{\mu} \beta^{\mu}$ transforms non-trivially under spatial coordinate transformations, so one can achieve, locally at least, $\pi_{T}=1$ by a spatial diffeomorhism. The rescaled lapse is determined by the gauge condition $T=x^{0}$ and the equation for $T$,

$$
1=\dot{T}=\frac{\tilde{N}}{2 \rho^{2}} \pi_{T}=\frac{\tilde{N}}{2 \rho^{2}}
$$

This shows that in the gauge $T=x^{0}$, the rescaled lapse $\tilde{N}$ reduces to $2 \rho^{2}$ and depends accordingly (asymptotically) only on time, which implies that the equal-time slices in the gauge $T=x^{0}$ are the same as the equal-time slices of the pseudo-Gaussian gauge $(\tilde{N}=1)$ considered in the text. The difference between the two cooordinate systems is a mere space-independent relabeling of the time coordinate. [If one imposes the further spatial coordinate condition that $\sqrt{g}$ does not depend on the spatial coordinates, these slices are also the slices of a Gaussian coordinate system.]

There are thus two equivalent descriptions of the asymptotic evolution:

- the reduced description, in which the motion is at each spatial point a unit velocity "relativistic" billiard motion in hyperbolic space $\gamma^{\mu}$ with Hamiltonian (D.9);

- the unreduced description, in which the motion is a lightlike motion in the space of all the scale factors $\beta^{\mu}$, interrupted by collisions against the hyperplanes $w_{A}(\beta)=0\left(\Leftrightarrow w_{A}(\gamma)=0\right)$.

In the second description, the Minkowskian time $\left(\sim \sum \beta^{\mu}\right)$ between two collisions grows and there is, at each collision, a redshift of the momentum 
because the walls are receding. In the first, projected description, the walls are fixed, so that the (average) time between two collisions is constant, as well as the average change in the momenta conjugate to the reduced variables. To be more precise, the momenta $\tilde{\pi}^{\mu}$ conjugate to the $\gamma^{\mu}$ 's remain of order unity, while the momenta $\pi^{\mu} \sim \rho^{-1} \tilde{\pi}^{\mu}$ conjugate to the $\beta^{\mu}$ 's go to zero.

\section{Notes}

1. We have stressed that the symmetry, dominant gravitational and $p$ form walls are all timelike. This provides an important consistency check of the BKL picture. The only spacelike wall that we know of is the cosmological constant term $\Lambda g \sim \exp \left[-2\left(\sum_{i} \beta^{i}\right)\right]$. Depending on the initial conditions - which do indeed set the scales -, this wall either prevents the system to reach the BKL small volume regime (there could be a bounce like in the de Sitter solution) or does not prevent the collapse, in which case it does not affect the BKL picture since the cosmological potential $\Lambda g$ goes to zero as $g$ goes to zero.

2. Number of collisions Since the the Hamiltonian is asymptotically $T$-independent in the reduced description, the number of collisions per unit time $T$ is asymptotically constant. Hence, $T$ is a measure of the number of collisions. One has $g \sim t^{2}$ and $T \sim \ln \ln t$ so the number of collisions goes like $\ln \ln t$.

\section{References}

[A02] E. Anderson, "Strong-coupled relativity without relativity," arXiv:gr-qc/0205118.

[AR01] L. Andersson and A.D. Rendall, "Quiescent cosmological singularities," Commun. Math. Phys. 218, 479-511 (2001) [arXiv:grqc/0001047].

[B92] V. A. Belinsky, JETP Letters 56, 422 (1992).

[BK73] V.A. Belinskii and I.M. Khalatnikov, "Effect of scalar and vector fields on the nature of the cosmological singularity," Sov. Phys. JETP 36, 591-597 (1973). 
[BKL70] V.A. Belinskii, I.M. Khalatnikov and E.M. Lifshitz, "Oscillatory approach to a singular point in the relativistic cosmology," Adv. Phys. 19, 525 (1970);

[BKL72] V.A. Belinskii, I.M. Khalatnikov and E.M. Lifshitz, "Construction of a general cosmological solution of the Einstein equation with a time singularity", Sov. Phys. JETP 35, 838-841 (1972).

[BKL82] V.A. Belinskii, I.M. Khalatnikov and E.M. Lifshitz, "A general solution of the Einstein equations with a time singularity," Adv. Phys. 31, 639 (1982).

[B02] B. K. Berger, "Numerical Approaches to Spacetime Singularities," arXiv:gr-qc/0201056.

[BGIMW98] B. K. Berger, D. Garfinkle, J. Isenberg, V. Moncrief and M. Weaver, "The singularity in generic gravitational collapse is spacelike, local, and oscillatory," Mod. Phys. Lett. A 13, 1565 (1998) [arXiv:gr-qc/9805063].

[BRWN82] E. Bergshoeff, M. de Roo, B. de Wit and P. van Nieuwenhuizen, "Ten-Dimensional Maxwell-Einstein Supergravity, Its Currents, And The Issue Of Its Auxiliary Fields," Nucl. Phys. B 195, 97 (1982).

[BMG88] P. Breitenlohner, D. Maison and G. W. Gibbons, "FourDimensional Black Holes From Kaluza-Klein Theories," Commun. Math. Phys. 120, 295 (1988).

[BDV98] A. Buonanno, T. Damour and G. Veneziano, "Pre-big bang bubbles from the gravitational instability of generic string vacua," Nucl. Phys. B 543, 275 (1999) [arXiv:hep-th/9806230].

[CM83] G. F. Chapline and N. S. Manton, "Unification Of Yang-Mills Theory And Supergravity In Ten-Dimensions," Phys. Lett. B 120, 105 (1983).

[CB83] D. F. Chernoff and J. D. Barrow, Phys. Rev. Lett. 50, 134 (1983).

[C72] D.M. Chitre, Ph. D. thesis, University of Maryland, 1972. 
[CL96] N. J. Cornish and J. J. Levin, "The mixmaster universe is chaotic," Phys. Rev. Lett. 78, 998 (1997) [arXiv:gr-qc/9605029]; "The mixmaster universe: A chaotic Farey tale," Phys. Rev. D 55, 7489 (1997) [arXiv:gr-qc/9612066].

[CJLP99] E. Cremmer, B. Julia, H. Lu and C. N. Pope, arXiv:hepth/9909099.

[CJS78] E. Cremmer, B. Julia and J. Scherk, "Supergravity Theory In 11 Dimensions," Phys. Lett. B 76, 409 (1978).

[DdBHS02] T. Damour, S. de Buyl, M. Henneaux and C. Schomblond, "Einstein billiards and overextensions of finite-dimensional simple Lie algebras," arXiv:hep-th/0206125.

[DH00a] T. Damour and M. Henneaux, "Chaos in superstring cosmology," Phys. Rev. Lett. 85, 920 (2000) [arXiv:hep-th/0003139]; [See also short version in Gen. Rel. Grav. 32, 2339 (2000).]

[DH00b] T. Damour and M. Henneaux, "Oscillatory behaviour in homogeneous string cosmology models," Phys. Lett. B 488, 108 (2000) [arXiv:hep-th/0006171].

[DH01] T. Damour and M. Henneaux, "E(10), BE(10) and arithmetical chaos in superstring cosmology," Phys. Rev. Lett. 86, 4749 (2001) [arXiv:hep-th/0012172].

[DHJN01] T. Damour, M. Henneaux, B. Julia and H. Nicolai, "Hyperbolic Kac-Moody algebras and chaos in Kaluza-Klein models," Phys. Lett. B 509, 323 (2001) [arXiv:hep-th/0103094].

[DHN02] T. Damour, M. Henneaux and H. Nicolai, "E(10) and a 'small tension expansion' of M theory," arXiv:hep-th/0207267.

[DHRW02] T. Damour, M. Henneaux, A. D. Rendall and M. Weaver, "Kasner-like behaviour for subcritical Einstein-matter systems," arXiv:gr-qc/0202069, to appear in Ann. Inst. H. Poincaré.

[D98] G. Dautcourt, "On the ultrarelativistic limit of general relativity," Acta Phys. Polon. B 29, 1047 (1998) [arXiv:gr-qc/9801093]. 
[DDRH88] J. Demaret, Y. De Rop and M. Henneaux, "Chaos In Nondiagonal Spatially Homogeneous Cosmological Models In Space-Time Dimensions > 10," Phys. Lett. B 211, 37 (1988).

[DHHST86] J. Demaret, J.L. Hanquin, M. Henneaux, P. Spindel and A. Taormina, "The Fate Of The Mixmaster Behavior In Vacuum Inhomogeneous Kaluza-Klein Cosmological Models," Phys. Lett. B 175, 129 (1986).

[DHS85] J. Demaret, M. Henneaux and P. Spindel, "Nonoscillatory Behavior In Vacuum Kaluza-Klein Cosmologies," Phys. Lett. 164B, 27 (1985).

[D67] B. S. Dewitt, "Quantum Theory Of Gravity. 1. The Canonical Theory," Phys. Rev. 160, 1113 (1967).

[E72] D. Eardley, E. Liang and R. Sachs, "Velocity-Dominated Singularities In Irrotational Dust Cosmologies," J. Math. Phys. 13, 99 (1972).

[EMcM93] A. Eskin and C. McMullen, "Mixing, counting and equidistribution in Lie groups", Duke Math. J. 71, 181-209 (1993).

[FF83] A.J. Feingold and I.B. Frenkel, Math. Ann. 263, 87 (1983)

[GV92] M. Gasperini and G. Veneziano, "Pre - big bang in string cosmology," Astropart. Phys. 1, 317 (1993) [arXiv:hep-th/9211021].

[GV02] M. Gasperini and G. Veneziano, "The pre-big bang scenario in string cosmology," arXiv:hep-th/0207130.

[HP70] S. W. Hawking and R. Penrose, "The Singularities Of Gravitational Collapse And Cosmology," Proc. Roy. Soc. Lond. A 314, 529 (1970).

[H78] S. Helgason, "Differential Geometry, Lie Groups, and Symmetric Scpaces", Graduate Studies in Mathematics vol. 34, American Mathematical Society, Providence 2001

[H79] M. Henneaux, "Geometry Of Zero Signature Space-Times," Print-79-0606 (Princeton), published in Bull. Soc. Math. Belg. 
31, 47 (1979) (note the misprints in the published version, absent in the preprint version).

[HPT82] M. Henneaux, M. Pilati and C. Teitelboim, "Explicit Solution For The Zero Signature (Strong Coupling) Limit Of The Propagation Amplitude In Quantum Gravity," Phys. Lett. B 110, 123 (1982).

[HM79] R. E. Howe and C. C. Moore, "Asymptotic properties of unitary representations," J. Functional Analysis 32, 72-96 (1979)

[IM01] G. Imponente and G. Montani, "On the Covariance of the Mixmaster Chaoticity," Phys. Rev. D 63, 103501 (2001) [arXiv:astro-ph/0102067].

[I76] C. J. Isham, Proc. Roy. Soc. Lond. A 351, 209 (1976).

[IKM94] V. D. Ivashchuk, A. A. Kirillov and V. N. Melnikov, JETP Lett. 60, N4 (1994).

[IM95] V.D. Ivashchuk and V.N. Melnikov, "Billiard Representation For Multidimensional Cosmology With Multicomponent Perfect Fluid Near The Singularity," Class. Quantum Grav. 12, 809 (1995).

[IM99] V. D. Ivashchuk and V. N. Melnikov, "Billiard representation for multidimensional cosmology with intersecting p-branes near the singularity," J. Math. Phys. 41, 6341 (2000) [arXiv:hepth/9904077].

[J86] R.T. Jantzen, "Finite-dimensional Einstein-Maxwell-scalar-field system," Phys. Rev. D 33, 2121-2135 (1986).

[J01] R. T. Jantzen, "Spatially homogeneous dynamics: A unified picture," arXiv:gr-qc/0102035.

[J80] B. Julia, LPTENS 80/16, Invited paper presented at Nuffield Gravity Workshop, Cambridge, Eng., Jun 22 - Jul 12, 1980.

[J85] B. Julia, in Lectures in Applied Mathematics, AMS-SIAM, vol 21 (1985), p.355. 
[Kac90] V.G. Kac, Infinite Dimensional Lie Algebras, 3rd edn., Cambridge University Press, 1990

[KM02] A. M. Khvedelidze and D. M. Mladenov, "Bianchi I cosmology and Euler-Calogero-Sutherland model," arXiv:gr-qc/0208037.

[K93] A. A. Kirillov, Sov. Phys. JETP 76, 355 (1993).

[KK87] A. A. Kirillov and A. A. Kochnev, JETP Letters 46, 436 (1987).

[KM94] A. A. Kirillov and V. N. Melnikov, "Dynamics Of Inhomogeneities Of Metric In The Vicinity Of A Singularity In Multidimensional Cosmology," Phys. Rev. D 52, 723 (1995) [grqc/9408004];

[KS00] A. A. Kirillov and G. V. Serebryakov, "Origin of a classical space in quantum cosmologies," Grav. Cosmol. 7, 211 (2001) [arXiv:hep-th/0012245].

[L97] V.G. LeBlanc, "Asymptotic states of magnetic Bianchi I cosmologies," Class. Quantum Grav. 14, 2281-2301 (1997); "Bianchi II magnetic cosmologies," Class. Quantum Grav. 15, 1607-1626 (1998).

[LWC00] J. E. Lidsey, D. Wands and E. J. Copeland, "Superstring cosmology," Phys. Rept. 337, 343 (2000) [arXiv:hep-th/9909061].

[LLK71] E. M. Lifshitz, I. M. Lifshitz and I. M. Khalatnikov, Sov. Phys. JETP 32, 173 (1971).

[LS01] U. Lindstrom and H. G. Svendsen, "A pedestrian approach to high energy limits of branes and other gravitational systems," Int. J. Mod. Phys. A 16, 1347 (2001) [arXiv:hep-th/0007101].

[LPS96] H. Lu, C. N. Pope and K. S. Stelle, "Weyl Group Invariance and p-brane Multiplets," Nucl. Phys. B 476, 89 (1996) [arXiv:hepth/9602140].

[Ma69] G. A. Margulis, "Applications of ergodic theory to the investigation of manifolds of negative curvature," Funct. Anal. Appl. 4, 335 (1969) 
[M69a] C.W. Misner, "Mixmaster universe," Phys. Rev. Lett. 22, 10711074 (1969).

[M69b] C. W. Misner, "Quantum Cosmology. 1," Phys. Rev. 186, 1319 (1969); "Minisuperspace," In *J $R$ Klauder, Magic Without Magic*, San Francisco 1972, 441-473.

[M94] C.W. Misner, in: D. Hobill et al. (Eds), Deterministic chaos in general relativity, Plenum, 1994, pp. 317-328 [gr-qc/9405068].

[MP95] R.V. Moody and A. Pianzola, Lie Algebras with Triangular Decomposition, Wiley, New York, 1995

[N92] H. Nicolai, "A Hyperbolic Lie Algebra From Supergravity," Phys. Lett. B 276, 333 (1992).

[OPR98] N. A. Obers, B. Pioline and E. Rabinovici, "M-theory and Uduality on $\mathrm{T}^{* *} \mathrm{~d}$ with gauge backgrounds," Nucl. Phys. B 525, 163 (1998) [arXiv:hep-th/9712084].

[R01] H. Ringström, "The Bianchi IX attractor," Ann. H. Poincaré 2, 405-500 (2001) [arXiv:gr-qc/0006035].

[R72a] M. P. Ryan, "The oscillatory regime near the singularity in Bianchi-type IX universes," Ann. Phys. (N.Y.) 70, 301 (1972).

[R72b] M. P. Ryan, "Hamiltonian cosmology," Springer-Verlag, Heidelberg (1972).

[RS75] M. P. Ryan and L. C. Shepley, "Homogeneous Relativistic Cosmologies," Princeton, USA: Univ. Pr. (1975) 320 P. ( Princeton Series In Physics).

[T78] C. Teitelboim, "The Hamiltonian Structure Of Space-Time," PRINT-78-0682 (Princeton), in: General Relativity and Gravitation, vol 1, A. Held ed., Plenum Press, 1980.

[W02] D. Wands, "String-inspired cosmology," Class. Quant. Grav. 19, 3403 (2002) [arXiv:hep-th/0203107]. 
[W99] M. Weaver, "Dynamics of magnetic Bianchi $\mathrm{VI}_{0}$ cosmologies," Class. Quantum Grav. 17, 421-434 (2000) [arXiv:grqc/9909043].

[Z84] R. Zimmer, Ergodic Theory and Semisimple Groups, (Birkhauser, Boston, 1984) 